\title{
AN ARITHMETIC ZARISKI PAIR OF LINE ARRANGEMENTS WITH NON-ISOMORPHIC FUNDAMENTAL GROUP
}

\author{
ENRIQUE ARTAL BARTOLO, JOSÉ IGNACIO COGOLLUDO-AGUSTÍN, BENOÎT GUERVILLE-BALLÉ, \\ AND MIGUEL MARCO-BUZUNÁRIZ
}

\begin{abstract}
In a previous work, the third named author found a combinatorics of line arrangements whose realizations live in the cyclotomic group of the fifth roots of unity and such that their non-complex-conjugate embedding are not topologically equivalent in the sense that they are not embedded in the same way in the complex projective plane. That work does not imply that the complements of the arrangements are not homeomorphic. In this work we prove that the fundamental groups of the complements are not isomorphic. It provides the first example of a pair of Galois-conjugate plane curves such that the fundamental groups of their complements are not isomorphic (despite the fact that they have isomorphic profinite completions).
\end{abstract}

\section{INTRODUCTION}

The relationship between topology and combinatorics is an important aspect in the study of hyperplane arrangements. As the main positive result, we have the fact that for a hyperplane arrangement (say, in a complex projective space), the cohomology ring of the complement is determined by the combinatorial data [18. In 1994 G. Rybnikov [20] found a pair of line arrangements in $\mathbb{P}^{2} \equiv \mathbb{P}^{2}(\mathbb{C})$ such that their fundamental groups are not isomorphic. In the long period between the announcement and the publication of the result, Rybnikov's statement was reproved by three of the authors of this paper (with J. Carmona) in [7, using group extensions involving the Alexander invariant of the group and a combinatorial generalization of some Rybnikov's ideas by the fourth named author [17. Note that Rybnikov's arrangements cannot be the complexification of a real arrangement.

Some years later, a new example of arrangements sharing the combinatorics but not all of the topological properties was found [ [6]. This example was weaker than Rybnikov's one in some aspects and somewhat stronger in other ones. The author distinguished the two arrangements using a braid monodromy invariant developed in [4; this invariant is able to distinguish two pairs formed by the complex projective plane and an algebraic curve but it does not give further information on the topology of the complement (in particular, on the fundamental group). These

2010 Mathematics Subject Classification. 14N20, 32S22, 14F35, 14H50, 14F45,14G32.

Key words and phrases. Line arrangements, Zariski pairs, number fields, fundamental group.

First, second and fourth authors are partially supported by MTM2013-45710-C2-1-P and MTM2016-76868C2-2-P. First and second authors are also partially supported by Grupo Geometría of Gobierno de Aragón/Fondo Social Europeo. Third author is partially supported by JSPS postdoctoral grant. 
arrangements are defined over $\mathbb{Q}(\sqrt{5})$ (in particular they are the complexification of a real arrangement) and, moreover, they have Galois-conjugate equations in this field. As a consequence the fundamental groups of their complements have the same profinite completion and they share all the topological properties of algebraic nature (quite a lot!).

Recently, the third named author [16] found another combinatorics with distinct topological realizations. He used a new invariant developed in [3, 9, computing the image of some special homological cycle in the complement by some associated character; this computation is doable using the injection of the boundary of a regular neighborhood of the arrangement in the complement, done in [14]. In this case the realizations are defined over the cyclotomic group of the fifth roots of unity and only the topology of the pairs is distinguished by this invariant as in the latter example. Up to our knowledge, these are (essentially) the only known examples of line arrangements with the same combinatorics and distinct topology.

The main result of this paper is that in fact, the fundamental groups of the realizations of the above combinatorics are not isomorphic (of course, for the realizations which are not complexconjugate). We have used the same method which was already successful in [7]. The notion of combinatorics can be generalized for arbitrary plane algebraic curves, see 2]: a pair of curves with the same combinatorics and distinct topology is called a Zariski pair. A Zariski pair is said to be arithmetic if its members have Galois-conjugate equations over a number field. As we stated before, it is hard to check if a Zariski pair candidate is an actual Zariski pair; as an example there are arithmetic Zariski pairs candidate communicated to the authors by Fan for which the known invariants failed to check if they are actual arithmetic Zariski pairs.

The arithmetic Zariski pairs are related to conjugate varieties. The fact that conjugate varieties may have distinct topology was proved by Serre [21]; other examples were found by Abelson [1] and more recently by many authors [10, 19, 15]. For the case of curves, the first example of an arithmetic Zariski pair is in [5] (a curve of degree 12). The complete list of such Zariski pairs for sextics was done by Shimada 22, 23, a longer list (replacing homeomorphism by a special type of diffeomorphism) was given by Degtyarev [12]. In all these examples, nothing is said about the topology of the complement; in fact, in 8 a Zariski pair with homeomorphic complements is given. Hence, our example is the first arithmetic Zariski pair with non-isomorphic fundamental group.

The paper is organized as follows. In $\$ 1$, we introduce the combinatorics $\mathscr{G}_{91}$ studied in the paper. In $\$ 2$, we study the homological rigidity of this combinatorics. The fact that $\mathscr{G}_{91}$ is homologically rigid and has no non-trivial automorphism implies that given two realizations $\mathcal{A}_{i}$, $i=1,2$, any isomorphism $\Phi: \pi_{1}\left(\mathbb{P}^{2} \backslash \mathcal{A}_{1}\right) \rightarrow \pi_{1}\left(\mathbb{P}^{2} \backslash \mathcal{A}_{2}\right)$ is \pm the identity in homology. In $\S 3$, we introduce the truncated Alexander invariants. In $\$$, we compute the fundamental groups using the wiring diagrams computed in [16] and we explain how to compute the truncated Alexander invariants. We show that no isomorphism $\Phi: \pi_{1}\left(\mathbb{P}^{2} \backslash \mathcal{A}_{1}\right) \rightarrow \pi_{1}\left(\mathbb{P}^{2} \backslash \mathcal{A}_{2}\right)$ can induce the identity on homology and using a conjugate arrangement, we do the same for its opposite, proving the result. Finally, the Sagemath code that carries out the heavy computations is outlined 
and explained in Appendix A. This code is available at https://github.com/enriqueartal/ ZariskiPair12Lines.git, see the README.md file inside for the details.

\section{ACKNowledgement}

The authors want to thank the anonymous referees for their suggestions that have helped in the exposition of this paper.

\section{Combinatorics And Realizations}

In this section a line combinatorics will be described together with several possible realizations on the complex projective plane.

\subsection{Line combinatorics.}

For the sake of completeness we will recall the definitions of line combinatorial types.

Definition 1.1. A line combinatorial type (or simply a (line) combinatorics) is a pair $\mathscr{C}:=$ $(\mathcal{L}, \mathcal{P})$, where $\mathcal{L}$ is a finite set and $\mathcal{P} \subset \mathcal{P}(\mathcal{L})$, satisfying:

(1) For all $P \in \mathcal{P}, \# P \geq 2$;

(2) For any $L_{1}, L_{2} \in \mathcal{L}, L_{1} \neq L_{2}, \exists ! P \in \mathcal{P}$ such that $L_{1}, L_{2} \in P$.

An ordered combinatorial type $\mathscr{C}^{\text {ord }}$ is a combinatorial type where $\mathcal{L}$ is an ordered set.

Notation 1.2. Given a combinatorial type $\mathscr{C}$, the multiplicity $m_{P}$ of $P \in \mathcal{P}$ is the number of elements $L \in \mathcal{L}$ such that $L \in P$; note that $m_{P} \geq 2$. In order to mimic the scenario where the combinatorics comes from a line arrangement, we will write $P \in L$ when the line $L$ is in the point $P$; this relation will also be indicated as $L<P$.

\subsection{The combinatorics $\mathscr{G}_{91}$.}

The combinatorics (and realizations) described here were originally presented in [16]. In what follows, a more geometrically insightful approach will be presented. This will be helpful in order to describe the required invariants to compare their fundamental groups.

Let us begin with an ordered set of four points $P_{1}, \ldots, P_{4} \in \mathbb{P}^{2}$ in general position (red points in Figure 1. Note that such a set is unique up to projective automorphism:

$$
P_{1}, \ldots, P_{4}=[1: 1: 1],[1:-1: 1],[1:-1:-1],[1: 1:-1] .
$$

The first four lines of our arrangement are the lines $L_{1}, \ldots, L_{4}$ of the quadrangle defined by $P_{1}, \ldots, P_{4}$ :

$$
L_{1}: x-z=0, \quad L_{2}: x+y=0, \quad L_{3}: x+z=0, \quad L_{4}: x-y=0 .
$$

The two diagonals of the quadrangle should also be considered: $L_{5}$ is the line $\overline{P_{1} P_{3}}$ and $L_{6}$ is the line $\overline{P_{2} P_{4}}$. Their equations are:

$$
L_{5}: y-z=0, \quad L_{6}: y+z=0 .
$$

After choosing two points $P_{5}$ (resp. $\left.P_{6}\right)$ on $L_{5}$ (resp. $L_{6}$ ) one can consider a set of four lines $L_{9}:=\overline{P_{1} P_{6}}, L_{10}:=\overline{P_{2} P_{5}}, L_{7}:=\overline{P_{3} P_{6}}, L_{8}:=\overline{P_{4} P_{5}}$ as shown in Figure 1. The intersection of 
these lines with the original lines $L_{1}, \ldots, L_{4}$ defines a set of points as follows: $Q_{i}=L_{i} \cap L_{i+6}$, $i=1, \ldots, 4$.

In principle, the newly defined points $Q_{1}, \ldots, Q_{4}$ need not be aligned. The combinatorics $\mathscr{G}_{91}^{\prime}$ is determined by a particular choice of $P_{5}$ and $P_{6}$ for which $Q_{1}, \ldots, Q_{4}$ belong in a line, say $L_{11}$.

The existence of such points $P_{5}$ and $P_{6}$ in $\mathbb{P}^{2}$ satisfying the condition given in $\mathscr{G}_{91}^{\prime}$ is not unique (as it was proved in [16]). In fact, there are four choices leading to the following realizations:

$$
\begin{aligned}
& L_{9}: x+\left(\xi^{2}+\xi\right) y+\overline{\left(\xi^{2}+\xi\right)} z=0, \quad L_{10}: x-\left(\bar{\xi}^{2}+\xi\right) y+\left(\bar{\xi}+\xi^{2}\right) z=0, \\
& L_{7}: x-\overline{\left(\xi^{2}+\xi\right)} y-\left(\xi^{2}+\xi\right) z=0, \quad L_{8}: x+\left(\bar{\xi}+\xi^{2}\right) y-\left(\bar{\xi}^{2}+\xi\right) z=0,
\end{aligned}
$$

where $\xi$ is a primitive fifth-root of unity.

Finally, one can also obtain an equation of the line $L_{11}$ joining $Q_{1}, \ldots, Q_{4}$ :

$$
L_{11}: 5 x+\left(1+2 \xi+3 \xi^{2}-\bar{\xi}^{2}\right) y-\left(2+4 \xi+\xi^{2}+3 \bar{\xi}^{2}\right) z=0 .
$$

For further use, the four realizations parametrized by the primitive fifth-roots of unity will be denoted by $\left(\mathcal{A}^{\prime}\right)^{\xi}$.

The ordered combinatorics $\mathscr{G}_{91}^{\prime}$ has a non-trivial automorphism group, generated by the product of the cyclic permutations of $\left(L_{1}, \ldots, L_{4}\right)$ and $\left(L_{7}, \ldots, L_{10}\right)$, and the transposition of $\left(L_{5}, L_{6}\right)$, while $L_{11}$ is invariant. Note that the action of this group of automorphisms can be realized by projective transformations sending cyclically $\left(\mathcal{A}^{\prime}\right)^{\xi^{i}}$ to $\left(\mathcal{A}^{\prime}\right)^{\xi^{i+1}}$.

The final combinatorics $\mathscr{G}_{91}$ with trivial automorphism group can be obtained from $\mathscr{G}_{91}^{\prime}$ by adding a new line $L_{12}$ joining $P_{1}$ and $Q_{2}$. The four realizations of $\mathscr{G}_{91}$ will be denoted by $\mathcal{A}^{\xi}$. The line $L_{12}$ has an equation:

$$
L_{12}: x-(1+\bar{\xi}) y+\bar{\xi} z=0 .
$$

In terms of Definition 1.1, the combinatorics is defined $\mathcal{L}=\{1, \ldots, 12\}$ and $\mathcal{P}$ below:

$$
\begin{gathered}
\{1,4,5,9,12\},\{1,2,6,10\},\{2,3,5,7\},\{3,4,6,8\},\{2,8,11,12\},\{1,7,11\},\{3,9,11\},\{4,10,11\} \\
\{5,8,10\},\{6,7,9\},\{5,6\},\{5,11\},\{6,11\},\{1,3\},\{2,4\},\{1,8\},\{2,9\},\{3,10\},\{4,7\} \\
\{7,8\},\{7,10\},\{8,9\},\{9,10\},\{3,12\},\{6,12\},\{7,12\},\{10,12\}
\end{gathered}
$$

\section{Homological Rigidity}

Let $\mathscr{C}=(\mathcal{L}, \mathcal{P})$ be a line combinatorics as presented in $\$ 1$. Let $\mathbb{Z}^{\mathcal{L}}$ be a free abelian group with basis $\left\{x_{L} \mid L \in \mathcal{L}\right\}$; the dual basis in $\left(\mathbb{Z}^{\mathcal{L}}\right)^{*}$ is denoted by $\left\{y_{L} \mid L \in \mathcal{L}\right\}$. We define $H_{1}=H_{1}^{\mathscr{C}}$ as the quotient of $\mathbb{Z}^{\mathcal{L}}$ by the principal submodule generated by $\sum_{L \in \mathcal{L}} x_{L}$; in the same way we denote its dual by $H^{1}=H_{\mathscr{C}}^{1}$. Note that $H^{1}$ is the kernel of the augmentation morphism $\varepsilon:\left(\mathbb{Z}^{\mathcal{L}}\right)^{*} \rightarrow \mathbb{Z}$ associated to the above basis. These lattices are naturally isomorphic to the first homology and cohomology groups of the complement in $\mathbb{P}^{2}$ of any realization of $\mathscr{C}$.

To avoid notation overload the class of $x_{L}$ in $H_{1}$ is still denoted by $x_{L}$. For each $P \in \mathcal{P}$, let us denote $x_{P}:=\sum_{L<P} x_{L}$. Let $H_{2}:=H_{2}^{\mathscr{C}}$ the subgroup of $H_{1} \wedge H_{1}$ generated by

$$
\left\{x_{L, P}:=x_{L} \wedge x_{P} \mid L<P \in \mathcal{P}\right\}
$$




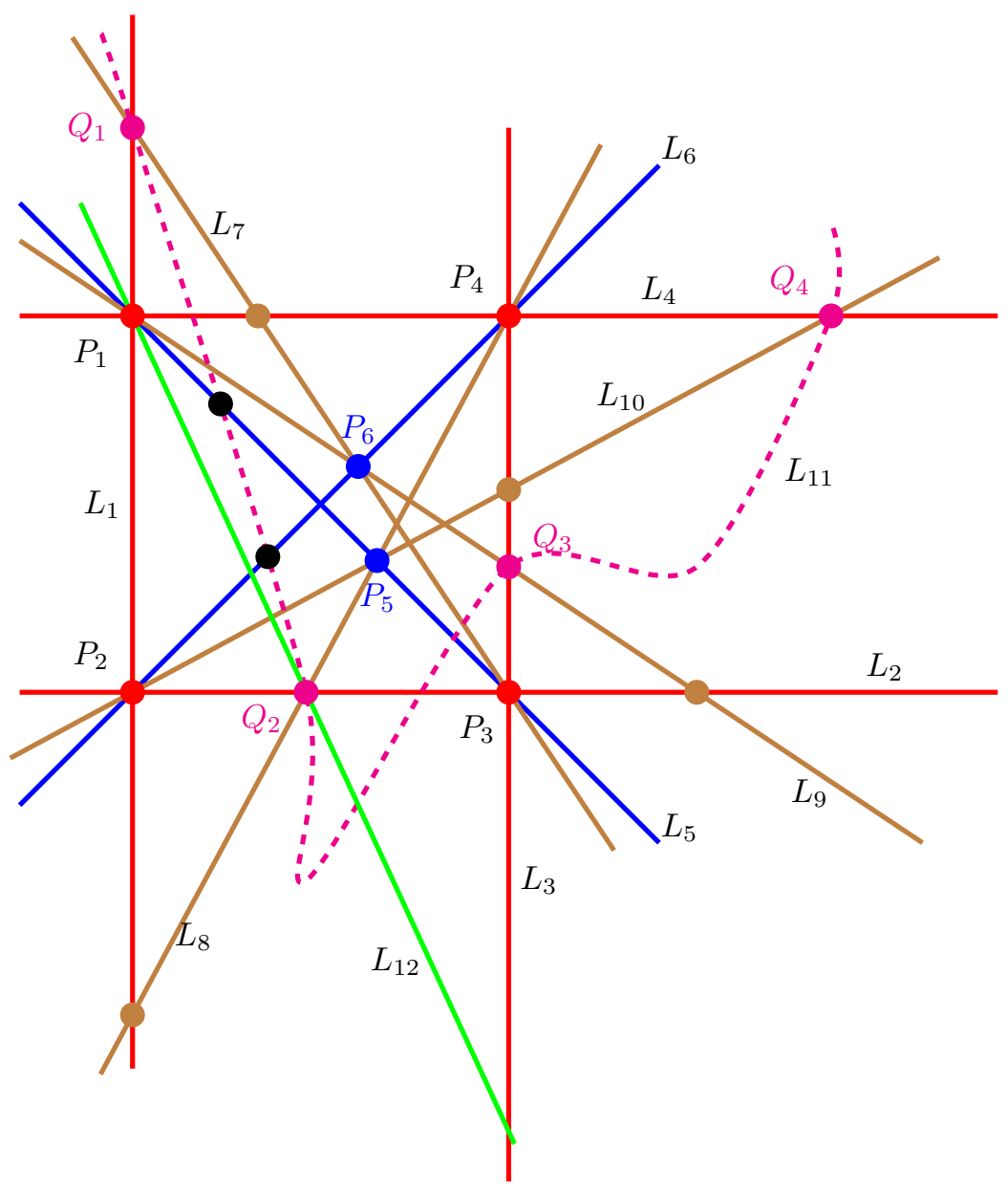

FigURE 1 . The combinatorics $\mathscr{G}_{91}$.

Its dual $H^{2}:=H_{\mathscr{C}}^{2}$ is the quotient of $H^{1} \wedge H^{1}$ by the subgroup generated by

$$
\left\{y_{L} \wedge y_{L^{\prime}}+y_{L^{\prime}} \wedge y_{L^{\prime \prime}}+y_{L^{\prime \prime}} \wedge y_{L} \mid L, L^{\prime}, L^{\prime \prime}<P \in \mathcal{P}\right\}
$$

as part of the Orlik-Solomon algebra. As above, these groups are naturally isomorphic to the second homology and cohomology groups of the complement in $\mathbb{P}^{2}$ of any realization of $\mathscr{C}$. Besides the automorphisms of the combinatorics, there are some geometrical automorphisms that will be considered here and play an essential role in this theory.

Definition 2.1. With the previous notation, any automorphism of $H_{1}^{\mathscr{C}}$ inducing a morphism of $H_{2}^{\mathscr{C}}$ will be referred to as an admissible automorphism of the combinatorics. The group of admissible automorphisms will be denoted by $\operatorname{Adm}_{\mathscr{C}}$.

Remark 2.2. Admissible automorphisms are also closely related to isomorphisms of fundamental groups of realizations of combinatorics. It is a consequence of Remark 3.9 that if $\mathcal{A}_{1}, \mathcal{A}_{2}$ are 
two realizations of $\mathscr{C}$ and $\varphi: \pi_{1}\left(\mathbb{P}^{2} \backslash \mathcal{A}_{1}\right) \rightarrow \pi_{1}\left(\mathbb{P}^{2} \backslash \mathcal{A}_{2}\right)$ is an isomorphism, under the above identifications, then its induced automorphism $\varphi_{*}: H_{1}^{\mathscr{C}} \rightarrow H_{1}^{\mathscr{C}}$ is admissible.

Definition 2.3 ([17]). A combinatorics $\mathscr{C}$ is called homologically rigid if $\operatorname{Adm}_{\mathscr{C}}= \pm 1_{H_{1}^{\mathscr{C}}} \times \operatorname{Aut}_{\mathscr{C}}$.

Homological rigidity is fundamental for the study of certain fine invariants of the topology of different realizations of $\mathscr{C}$. Checking the homological rigidity of a combinatorics is a subtle combinatorial property.

The main tools for the study of admissible automorphisms and homological rigidity are the strata of a certain stratification of $H_{\mathscr{C}}^{1}$ called resonance varieties, which will be described next.

\subsection{The resonance varieties of a combinatorics.}

For any $\omega \in H^{1}=H_{\mathscr{C}}^{1}$, one can define a complex $\left(H^{\bullet}, \wedge \omega\right)$ given by the wedge product as follows:

$$
\begin{aligned}
& H^{0}=\mathbb{Z} \stackrel{\wedge \omega}{\longrightarrow} H^{1} \stackrel{\wedge \omega}{\longrightarrow} H^{2} \quad \rightarrow 0 \\
& \sigma \mapsto \sigma \wedge \omega .
\end{aligned}
$$

We are interested in the first cohomology of this complex $H^{1}\left(H^{\bullet}, \wedge \omega\right)$.

Definition 2.4. The $k$-th resonance variety of $\mathscr{C}$ is defined as

$$
\mathcal{R}_{k, \mathscr{C}}:=\left\{\omega \in H^{1} \mid \operatorname{rank} H^{1}\left(H_{\mathscr{C}}^{\bullet}, \wedge \omega\right) \geq k\right\} \subset H^{1} .
$$

Analogously, one can define $\mathcal{R}_{k, \mathscr{C}, \mathbb{K}}$ over a field $\mathbb{K}$ when considering the complex over $\mathbb{K}$ and stratifying the vector space $H^{1} \otimes \mathbb{K}$ by the dimension of the associated cohomology.

As expected, the resonance varieties $\mathcal{R}_{k, \mathscr{C}, \mathbb{K}}$ of a combinatorics coincide with the resonance varieties over $\mathbb{K}$ of any realization of $\mathscr{C}$.

Each $k$-th resonance component over $\mathbb{Z}$ is determined by a subcombinatorics with a certain structure called combinatorial pencil (cf. [17]) or multinet (cf. [13]) with $k+2$ fibers. The structure of such combinatorial pencils with $k+2$ fibers mimics the combinatorial properties of a line arrangement which is the union of $k+2$ fibers of a pencils of curves. Such subarrangements will be referred to here using the typical pencil they describe. For instance multiple point, Cevatype (the combinatorics of the six lines in a generic pencil of smooth conics) or Hesse type (for the combinatorics of the 12 lines in an arrangement of smooth cubics based at their 9 inflection points).

Let us denote by $S$ a certain subcombinatorics of $\mathscr{C}$ (obtained as a subset of lines and their incidence relations) forming a combinatorial pencil. There is exactly one irreducible component in the resonance varieties of $S$ which is not contained in the resonance varieties of a subcombinatorics. Such component will be denoted by $H_{S}$ and will be referred to as the resonance component associated to $S$. Note that its dimension is $k+1$, if $k+2$ is the number of fibers of the combinatorial pencil.

Definition 2.5. Three subcombinatorics $S_{1}, S_{2}, S_{3}$ of combinatorial pencils in a line combinatorics are said to form a triangle if

$$
\operatorname{codim} \bigcap_{i} H_{S_{i}}=\sum_{i} \operatorname{codim} H_{S_{i}}-1
$$


The following result is obtained in [17, Lemma 6].

Lemma 2.6. Let $\rho \in A d m_{\mathscr{C}}$ be an admissible automorphism of $H_{1}$. Then its dual $\rho^{*}$ preserves triangles.

As depicted in Lemma 2.6, the resonance components (their dimension and the number of triangles they belong to) can impose conditions on admissible automorphisms. The following is an immediate consequence of [25].

Corollary 2.7. Let $\mathscr{C}$ be a realizable combinatorics and $R$ a $k$-th resonance component with $k>2$. Then $R$ is associated with the combinatorial pencil of a multiple point of multiplicity $m=$ $k+2>4$.

This has the following consequence on an admissible automorphism $\rho$. Let $P_{m}$ be a point on $\mathcal{P}$ of multiplicity $m$, which is a subcombinatorics of $\mathscr{C}$ and denote by $H_{P_{m}} \subset H^{1}$ its associated resonance component of dimension $k$. Then any admissible automorphism $\rho: H_{1} \rightarrow H_{1}$ must be such that $\rho^{*}\left(H_{P_{m}}\right)=H_{Q_{m}}$ for another $Q_{m} \in \mathcal{P}$ of multiplicity $m$.

This results in certain combinatorial conditions on the images of the special basis in $H_{1}$. If enough of these conditions concur, one can eventually be able to state homological rigidity. The idea behind this concept comes from the work of Rybnikov (cf. [20, 7]).

\subsection{The homological rigidity of $\mathscr{G}_{91}$.}

In our example, it is possible to compute the combinatorial pencils contained in the combinatorics $\mathscr{G}_{91}$ described in $\$ 1$ the 10 multiple points and 15 Ceva-type subarrangements. Only one of the 25 combinatorial pencils has 5 fibers (the quintuple point).

The number of triangles $\Delta_{S}$ in a given subcombinatorics $S$ as well as the number of triangles $\Delta_{P_{1}, S}$ containing the quintuple point $P_{1}$ are recorded in Table 1 .

Note that the subcombinatorics $S_{i}, i=1, \ldots, 5$ correspond to triple points, whereas $S_{i}$, $i=6, \ldots, 9$ correspond to quadruple points, and $S_{10}$ is the combinatorics of the quintuple point. Finally, the remaining $S_{i}, i=11, \ldots, 25$ correspond to the combinatorics of Ceva-type arrangements.

As a consequence of Table 1 one has the following.

Proposition 2.8. The realizable combinatorics $\mathscr{G}_{91}$ from $\S 1$ is homologically rigid. Moreover, the only admissible automorphisms of $H_{\mathscr{G}_{91}}^{1}$ are $\pm 1_{H_{\mathscr{G}_{91}}^{1}}$.

Proof. By Lemma 2.6 and Corollary 2.7. triangles containing the quintuple point are also preserved. Since no subcombinatorics of dimension 3 (the quadruple points) are equal in both the last two columns, such resonance components are invariant by $\rho$. Analogously, resonance components of triple points are also $\rho$-invariant. We use this information to prove the property. 


\begin{tabular}{|c|c|c|c|c|}
\hline$i$ & subcombinatorics $S_{i}$ & $\operatorname{dim} H_{S}$ & $\Delta_{S}$ & $\Delta_{S, P_{1}}$ \\
\hline 1 & $1,7,11$ & 2 & 18 & 7 \\
2 & $3,9,11$ & 2 & 22 & 8 \\
3 & $4,10,11$ & 2 & 21 & 7 \\
4 & $5,8,10$ & 2 & 24 & 7 \\
5 & $6,9,7$ & 2 & 16 & 6 \\
6 & $1,2,6,10$ & 3 & 53 & 12 \\
7 & $2,3,5,7$ & 3 & 49 & 13 \\
8 & $2,8,11,12$ & 3 & 57 & 15 \\
9 & $4,3,6,8$ & 3 & 50 & 12 \\
10 & $1,4,5,9,12$ & 4 & 91 & 91 \\
11 & $1,2,3,4,5,6$ & 2 & 24 & 8 \\
12 & $1,2,4,6,8,12$ & 2 & 24 & 8 \\
13 & $1,2,4,10,11,12$ & 2 & 20 & 7 \\
14 & $1,2,5,6,7,9$ & 2 & 14 & 7 \\
15 & $1,2,5,7,11,12$ & 2 & 14 & 7 \\
16 & $1,2,5,8,10,12$ & 2 & 20 & 8 \\
17 & $1,3,5,7,9,11$ & 2 & 14 & 7 \\
18 & $1,4,5,6,8,10$ & 2 & 19 & 6 \\
19 & $2,3,4,5,8,12$ & 2 & 20 & 8 \\
20 & $2,3,5,6,8,10$ & 2 & 14 & 0 \\
21 & $2,3,5,9,11,12$ & 2 & 18 & 9 \\
22 & $2,4,6,8,10,11$ & 2 & 15 & 0 \\
23 & $3,4,5,6,7,9$ & 2 & 12 & 6 \\
24 & $3,4,8,9,11,12$ & 2 & 13 & 7 \\
25 & $4,5,8,10,11,12$ & 2 & 15 & 7 \\
\hline
\end{tabular}

TABLE 1. Combinatorics of triangles in $\mathscr{G}_{91}$

Let $\rho: H_{1} \rightarrow H_{1}$ be an admissible automorphism. Let us choose any lift $\tilde{\rho}$ fitting in the following commutative diagram

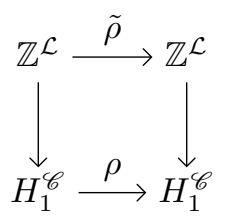

Let $A$ be a matrix of $\tilde{\rho}$ in the natural basis. Note that the columns of $A$ are only well defined up to addition of a multiple of $v:=(1, \ldots, 1)$ and that ${ }^{t} A$ is the matrix of a lift of $\rho^{*}$. Let $\left(i_{1}, \ldots, i_{r}\right)$ be a multiple point. Since $\rho^{*}$ fixes the resonance component associated to this point, with basis $y_{i_{2}}-y_{i_{1}}, \ldots, y_{i_{r}}-y_{i_{1}}$, we deduce the following fact. Consider the submatrix of $A$ 
given by the rows $i_{1}, \ldots, i_{r}$ and the columns distinct from $i_{1}, \ldots, i_{r}$; then, all the rows of this matrix are equal. This comes from the fact that $\rho^{*}\left(y_{i_{2}}-y_{i_{1}}\right)$ is in the subspace spanned by $y_{i_{1}}, \ldots, y_{i_{r}}$.

It is not hard to prove that after imposing the above condition, the matrix $A$ has the following property: each column has constant entries outside the diagonal. Since the matrix $A$ can be transformed by adding multiples of $v$ to the columns, we can assume that $A$ is diagonal.

Since $\rho$ is a group automorphism, one has $\rho\left(x_{L}\right)=\varepsilon_{L} x_{L}$, where $\varepsilon_{L} \in\{-1,1\}$. Moreover, the condition $\rho\left(\sum_{L} x_{L}\right)=0$ implies that all $\varepsilon_{L}$ are equal and the result follows.

\section{The truncated Alexander Invariants}

In this section the truncated Alexander invariants introduced in [7] will be recalled. Let $G$ be a group, and let $H=H(G):=G / G^{\prime}$ be its abelianization and consider $M=M(G):=G^{\prime} / G^{\prime \prime}$ as an abelian group. The conjugation action of $G$ on $G^{\prime}$, namely, $g \cdot h \mapsto g h g^{-1}$ induces an action of $H$ on $M$. This action extends by linearity to $\Lambda:=\mathbb{Z}[H]$, the group algebra of $H$. The Alexander invariant of $G$ is the abelian group $M$ together with the $\Lambda$-module structure.

Note that $\Lambda$ is, in general, not a PID and hence it is not easy to give complete invariants for the Alexander invariant. One standard way to approximate the structure of $M$ is by considering its truncation with respect to a special ideal. A standard way to do this is by means of $\mathfrak{m} \subset \Lambda$ the augmentation ideal of $\Lambda$, i.e. the kernel of the map $\Lambda \rightarrow \mathbb{Z}$ defined by $h \mapsto 1, \forall h \in H$ (despite the notation, note that $\mathfrak{m}$ is not a maximal ideal).

Definition 3.1. The truncated Alexander invariant of $G$ of order $k$ associated with $M=M(G)$ and the augmentation ideal $\mathfrak{m} \subset \Lambda$ is the quotient $M_{k}:=M / \mathfrak{m}^{k} M=M \otimes_{\Lambda} \Lambda / \mathfrak{m}^{k}, k \geq 1$, with its $\Lambda / \mathfrak{m}^{k}$-module structure.

These invariants are related to the Chen groups $\tilde{\gamma}_{k}(G)$ of $G$, that is, the lower central series of $G / G^{\prime \prime}$, the maximal metabelian quotient of $G$. They may alternatively be defined as $\tilde{\gamma}_{k+2}(G):=$ $\operatorname{ker}\left(\varphi_{k}: G^{\prime} \rightarrow M_{k}\right)$ where $\varphi_{k}$ is the quotient map.

Notation 3.2. Given two elements $p, q \in G^{\prime}$, the notation $p \stackrel{k}{=} q$ will be used meaning equality as elements in $M_{k}$, that is, $\varphi_{k}(p)=\varphi_{k}(q) \in M_{k}$.

Example 3.3. Let $\mathcal{A}$ be a line arrangement in $\mathbb{P}^{2}$, and let $G_{\mathcal{A}}:=\pi_{1}\left(\mathbb{P}^{2} \backslash \mathcal{A}\right)$. We will refer to $M(\mathcal{A}):=M\left(G_{\mathcal{A}}\right)$ (resp. $M_{k}(\mathcal{A}):=M_{k}\left(G_{\mathcal{A}}\right)$ ) as the Alexander invariant (resp. truncated Alexander invariants) of $\mathcal{A}$.

If $\mathcal{A}=\left\{L_{0}, L_{1}, \ldots, L_{\ell}\right\}$, then $G_{\mathcal{A}}$ admits a finite presentation

$$
G_{\mathcal{A}}=\left\langle x_{1}, \ldots, x_{\ell} \mid R_{1}, \ldots, R_{s}\right\rangle
$$

where $x_{i}$ is a meridian of the line $L_{i}$ and the words $R_{j}$ are commutators. Hence $H$ is a free abelian group of rank $\ell$, generated by the classes $t_{1}, \ldots, t_{\ell}$ of meridians $x_{1}, \ldots, x_{\ell}$ of the lines. As a consequence, $\Lambda=\mathbb{Z}\left[t_{1}^{ \pm 1}, \ldots, t_{\ell}^{ \pm 1}\right]$ is a ring of Laurent polynomials. The augmentation ideal $\mathfrak{m}$ is generated by the polynomials $\left\{t_{i}-1\right\}_{i=1}^{\ell}$. 
The Alexander invariant of $\mathcal{A}$ is generated as a $\Lambda$-module by $x_{i, j}, 1 \leq i<j \leq \ell$, the class of $\left[x_{i}, x_{j}\right]=x_{i} x_{j} x_{i}^{-1} x_{j}^{-1}$ in $M$. Note that

$$
\left[x_{i}, x_{j} x_{k}\right]=x_{i, j}+t_{j} x_{i, k} \bmod G^{\prime \prime} .
$$

This way, each relation $R_{k}$ induces a linear combination $\tilde{R}_{k}$ of $\left\{x_{i j}\right\}$ with coefficients in $\Lambda$. As was shown in [7, Proposition 2.8], the module $M(\mathcal{A})$ is the quotient of the free module generated by $x_{i, j}, 1 \leq i<j \leq \ell$, by the submodule generated by $\tilde{R}_{1}, \ldots, \tilde{R}_{s}$, and the so-called Jacobi relations

$$
\left(t_{i}-1\right) x_{j, k}-\left(t_{j}-1\right) x_{i, k}+\left(t_{k}-1\right) x_{i, j}, \quad 1 \leq i<j<k \leq \ell .
$$

Remark 3.4. Each multiple point $P$ produces $m(P)-1$ relations. These relations read in $M_{1}$ as follows. If $P$ is the intersection point of $L_{i_{1}}, \ldots, L_{i_{m}}$, then it produces

$$
\forall k \in\{2, \ldots, m\} \quad \sum_{j=1}^{m} x_{i_{k}, i_{j}} \stackrel{1}{=} 0 .
$$

Notation 3.5. We will denote $\sigma_{i}:=t_{i}-1$. Note that $\Lambda / \mathfrak{m}^{k}$ is isomorphic to $\mathbb{Z}\left[\sigma_{1}, \ldots, \sigma_{\ell}\right] / \mathfrak{a}^{k}$, where $\mathfrak{a}$ is the ideal generated by $\sigma_{1}, \ldots, \sigma_{\ell}$. The classes in $\Lambda / \mathfrak{m}^{k}$ are represented by polynomials in $\sigma_{1}, \ldots, \sigma_{\ell}$ of degree less than $k$. Note that the units are those polynomials whose degree zero coefficient equals \pm 1 .

Proposition 3.6 ([7, Proposition 2.15]). Let $\psi\left(X_{1}, \ldots, X_{m}\right)$ be a word in the letters $\left\{X_{1}, \ldots, X_{m}\right\}$. If $p_{i}, q_{i} \in G^{\prime}$ and $p_{i} \stackrel{k}{=} q_{i}(i=1, \ldots, m)$, then $\left[g, \psi\left(p_{1}, \ldots, p_{m}\right)\right] \stackrel{k+1}{\equiv}\left[g, \psi\left(q_{1}, \ldots, q_{m}\right)\right], \forall g \in G$. In particular, if $p \in M_{k}(\mathcal{A})$ then $[g, p]$ is a well-defined element of $M_{k+1}(\mathcal{A})$; if $g=x_{i}$ this element will be written as $\sigma_{i} p \in M_{k+1}(\mathcal{A})$.

Note that $M_{k}(\mathcal{A})$ is isomorphic (as an Abelian group) to the graduate group $\operatorname{gr}^{0} M_{k}(\mathcal{A}) \oplus$ $\ldots \oplus \mathrm{gr}^{k-1} M_{k}(\mathcal{A})$ by means of the morphism

$$
p \cdot x_{i, j} \mapsto p_{0} x_{i, j}+p_{1} x_{i, j}+\ldots+p_{k-1} x_{i, j},
$$

where $p$ is a polynomial in $\left\{\sigma_{1}, \ldots, \sigma_{r}\right\}$ and $p=p_{0}+\ldots+p_{k-1}$ is its homogeneous decomposition. Note that this isomorphism is not canonical, since it depends on the given set of generators of $G$.

For instance, any automorphism of $G$ that sends $x_{i}$ to $x_{i} \alpha_{i}$, (with $\alpha_{i} \in G^{\prime}$ ) induces an automorphism of $M_{k}(\mathcal{A})$ :

$$
\left[x_{i}, x_{j}\right] \mapsto\left[x_{i} \alpha_{i}, x_{j} \alpha_{j}\right] \stackrel{k}{=}\left[x_{i}, x_{j}\right]+t_{j} \sigma_{i} \alpha_{j}-t_{i} \sigma_{j} \alpha_{i} .
$$

Since this automorphism respects the filtration it also induces an automorphism of $\operatorname{gr} M_{k}(\mathcal{A})$. Note that the automorphism of $\operatorname{gr} M_{k}(\mathcal{A})$ it is always the identity but, in general, the automorphism of $M_{k}(\mathcal{A})$ is non-trivial.

Let us study the relationship of this invariants with combinatorics. Let us fix a combinatorics $\mathscr{C}$ and a realization $\mathcal{A}$ of $\mathscr{C}$. In $\$ 2$ we defined the free abelian module $H_{1}^{\mathscr{C}}$ generated by $x_{L}, L \in \mathcal{L}$, with the relation $\sum_{L \in \mathcal{L}} x_{L}=0$. Recall that the free abelian group $H_{\mathcal{A}}$ is generated 
also by the classes of positive meridians $g_{L}$ subject to the relation $\sum_{L \in \mathcal{L}} g_{L}=0$ and there is a natural isomorphism between $H_{1}^{\mathscr{C}}$ and $H_{\mathcal{A}}$. The composition $h: G_{\mathcal{A}} \rightarrow H_{\mathcal{A}} \rightarrow H_{1}^{\mathscr{C}}$

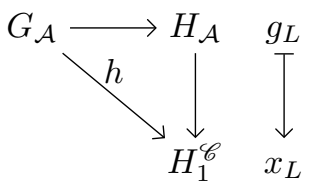

is a meridian structure of the group $G_{\mathcal{A}}$. A meridian $g_{L_{i}}$ has been denoted $x_{i}$ above; we will identify $x_{i}$ with $x_{L_{i}}$ without further notice.

Remark 3.7. Note that given a meridian structure $h$ in $G_{\mathcal{A}}$ it is in general not possible to recover the conjugacy classes of the meridians, only their homology classes are fixed.

We are going to define the naive truncated Alexander invariant $M_{2}^{\mathscr{C}}$ of the combinatorics $\mathscr{C}$. Let $H_{1}^{\mathscr{C}, \Lambda}:=H_{1}^{\mathscr{C}} \otimes_{\mathbb{Z}} \Lambda / \mathrm{m}^{2}$; then $M_{2}^{\mathscr{C}}$ is defined as the quotient of $H_{1}^{\mathscr{C}, \Lambda} \wedge H_{1}^{\mathscr{C}, \Lambda}$, with generators $x_{i, j}:=x_{i} \wedge x_{j}$, by a submodule generated by two sets, one coming from the combinatorics

$$
\sum_{j=1}^{m} x_{i_{j}, i_{k}}, \quad \forall P=\left\{L_{i_{1}}, \ldots, L_{i_{m}}\right\}, \forall k \in\{2, \ldots, m\},
$$

and another one coming from the Jacobi relations:

$$
\sigma_{i_{1}} x_{i_{2}, i_{3}}+\sigma_{i_{2}} x_{i_{3}, i_{1}}+\sigma_{i_{3}} x_{i_{1}, i_{2}}, \quad \forall L_{i_{1}}, L_{i_{2}}, L_{i_{3}} \in \mathcal{L} .
$$

From (3.4) we can forget the points $P$ such that $P \in L$ for some particular $L$. The graduate groups $\operatorname{gr}^{k} M_{2}^{\mathscr{C}}$ are defined accordingly for $k=0,1$. The following result is straightforward.

Proposition 3.8. For a realization $\mathcal{A}$, the graduate group $\operatorname{gr}^{k} M=\operatorname{gr}^{k} M_{2}, k=0,1$ is isomorphic to $\mathrm{gr}^{k} M^{\mathscr{C}}$. In particular, those graduate groups depend only on the combinatorics.

Remark 3.9. Note that the $\Lambda$-action on $\operatorname{gr}_{k} M_{2}^{\mathscr{C}}$ is reduced to the $\mathbb{Z}$-action. Moreover $\operatorname{gr}^{0} M_{2}^{\mathscr{C}} \equiv$ $M_{1}^{\mathscr{C}}$ coincides with $\left(H_{1}^{\mathscr{C}} \wedge H_{1}^{\mathscr{C}}\right) / H_{2}^{\mathscr{C}}$, compare (3.4) and 2.1). The analog result for $M_{k}$ is not true in general.

\section{Fundamental groups And the Alexander invariant isomorphism test}

In this section the main Alexander invariant homomorphism (isomorphism) test will be computed. In order to do so we will need to provide with a presentation of the fundamental groups of two realizations of the combinatorics $\mathscr{G}_{91}$. This information will allow us to give a presentation for the truncated Alexander invariants and finally we will prove the failure of the Alexander invariant isomorphism test. The homological rigidity of the combinatorics will allow for the Alexander invariant isomorphism test to be very close to an isomorphism test for fundamental groups. 


\subsection{Fundamental groups and braided wiring diagrams.}

Our purpose is to compare the fundamental groups $G^{i}$ of the complements $X_{i}:=\mathbb{P}^{2} \backslash \mathcal{A}^{\xi^{i}}$, $i=1, \ldots, 4$ of the different realizations of the combinatorics $\mathscr{G}_{91}$ as defined in $\S 1$. Note that $X_{i}$ and $X_{5-i}$ are homeomorphic via the conjugation automorphism $\mathbb{P}^{2} \rightarrow \mathbb{P}^{2}$, defined as $[x: y:$ $z] \mapsto[\bar{x}: \bar{y}: \bar{z}]$. Therefore $G^{i} \cong G^{5-i}$ and thus it only remains to study whether or not $G^{1}$ and $G^{2}$ are isomorphic. If we have fixed the meridian structure for each $G^{i}$ (associated to the realizations), the isomorphism $G^{i} \rightarrow G^{5-i}$ induces $-1_{H_{1}^{\mathscr{C}}}$ on $H_{1}^{\mathscr{C}}$.

Consider the realizations $\mathcal{A}^{\xi^{i}}, i=1,2$. In order to study the complements $X_{1}$ and $X_{2}$ we will use an adaptation of the Zariski-Van Kampen method to obtain a presentation of $G^{i}$. To begin with, one needs to project $\mathbb{P}^{2}$ from a point not on $\mathcal{A}^{\xi^{i}}$ and then obtain a system of braids, whose number of strings is the degree of the arrangement (i.e., the number of lines). The action of these braids on a free group will provide the required set of relations on the group. Our variation of the method allows for projections from a point on the arrangement, namely the point $P_{1}$, that is $\pi: \mathbb{P}^{2} \backslash\left\{P_{1}^{i}\right\} \rightarrow \mathbb{P}^{1}$ defined by $\pi(P)=\overline{P P_{1}^{i}}$, where $\mathbb{P}^{1}$ is identified with the space of lines in $\mathbb{P}^{2}$ passing through $P_{1}^{i}$. This causes a great deal of simplification since the braids obtained have as many strings less as the multiplicity of the chosen projection point in the arrangement.

In our situation the number of strings drops from 12, in the classical method, to 7. Note that $L_{1}^{i}, L_{4}^{i}, L_{5}^{i}, L_{9}^{i}$, and $L_{12}^{i}$ are points in the image of $\pi$. Moreover, denote by $T_{1}, \ldots, T_{4}$ the lines joining $P_{1}^{i}$ and the remaining double points of the arrangement (that is, points not on any line passing through $P_{1}^{i}$ ), also denote by $X_{i}:=\mathbb{P}^{2} \backslash \mathcal{A}^{\xi^{i}}$ the complement of the arrangement, and finally $\tilde{X}_{i}:=X_{i} \backslash \bigcup_{j=1}^{4} T_{j}$. Then $\left.\pi\right|_{\tilde{X}_{i}}$ is a locally trivial fibration whose fiber is $F:=$ $\mathbb{C} \backslash\{7$ points $\}$. Technically, if one blows up the point $P_{1}^{i}$, then this fibration can be extended to the exceptional divisor, which becomes a canonical section. This fibration can be understood from the action of the fundamental group of the image $\mathbb{P}^{1} \backslash\{9$ points $\}$ on the fiber $F$. This action is called the monodromy of the fibration and can be read off its braided wiring diagram.

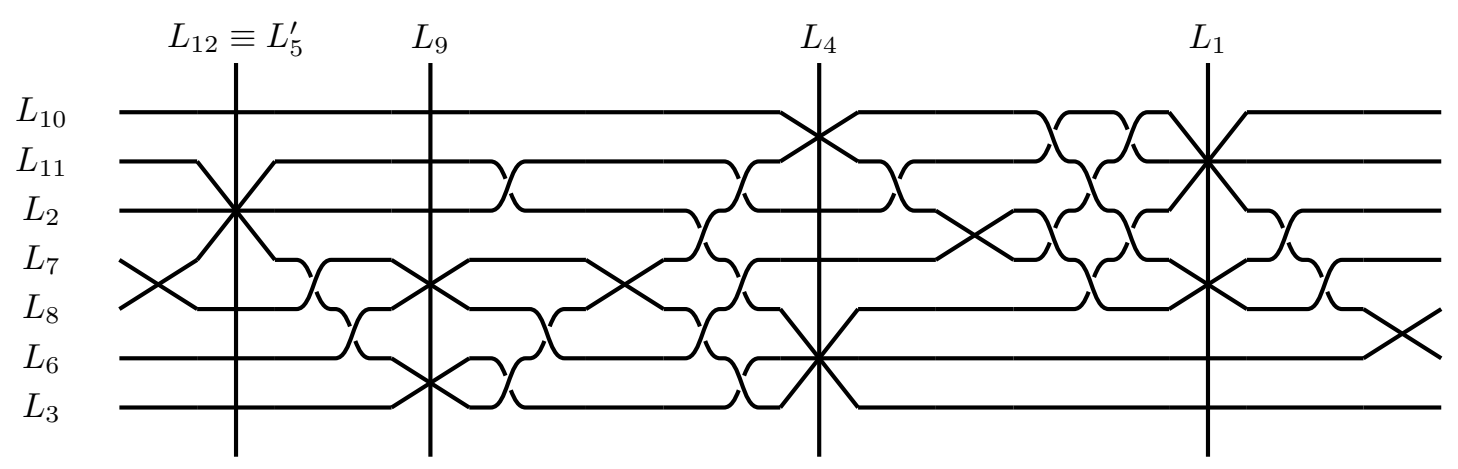

FiguRE 2. Wiring diagram of the arrangement $\mathcal{A}^{\zeta_{5}}$

In a nutshell, a braided wiring diagram describes the preimage in $\mathcal{A}^{\xi^{i}}$ by $\pi$ of a closed path $\gamma$ on the base $\mathbb{P}^{1}$ starting at a base point and going through all the points in the discriminant of $\pi$. This will be represented by a degenerated braid with crossings and multiple points (one 


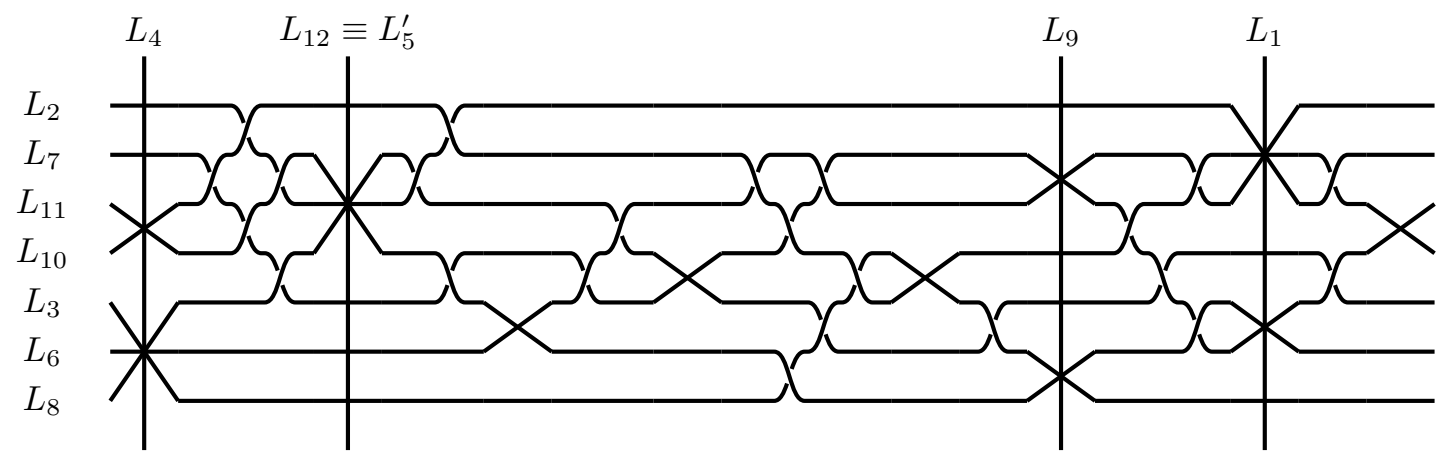

FiguRE 3. Wiring diagram of the arrangement $\mathcal{A}^{\zeta_{5}^{2}}$

multiple point for each singular point of the arrangement except for $\left.P_{1}^{i}\right)$. For more details, see [11. To the original definition of braided wiring diagram we have added the possibility of projecting from a singular point of the arrangement. This results in the vertical lines shown in the figures, corresponding to the degenerated fibers in the arrangement which have to be plotted too. Moreover, since the loop surrounding all the points on the discriminant is trivial in the fundamental group of the base, the closed path $\gamma$ could skip one point on the discriminant and still contain all the necessary information to recover the fibration.

Braided wiring diagrams for $\mathcal{A}^{\xi^{i}}, i=1,2$ as described above for paths skipping the image of the line $L_{5}^{i}$ are shown in Figures 2 and 3 . These plots can be obtained in a precise way, using the fact that lines can be parametrized.

In order to simplify the way of computing the fundamental groups, we perturb a little bit the projection point inside the line at infinity to obtain a wiring diagram without vertical lines, see [16]. We obtain a wiring diagram of 11 lines, where the former vertical lines start up in the left-hand side (the rightest the highest) and end below in the right-hand side. From these diagrams one obtains immediately the braid monodromy which allows to compute the fundamental group $G^{i}:=\pi_{1}\left(X_{i}\right)$ as follows. Let $\mu_{i}$ be a geometric basis of meridians generating the fundamental group of the fiber (in our case $\mathbb{F}_{11}=\pi_{1}(\mathbb{C} \backslash\{11$ points $\})$ ). The fundamental group $G^{i}$ is generated by $\left\{\mu_{k}\right\}_{k=1}^{11}$.

Let us explain how each point $Q$ on the discriminant of the projection $\pi$ induces a number of relations. The point $Q$ is associated to a multiple point involving $m_{Q}$ strands with indices $i_{Q}+1, \ldots, i_{Q}+m_{Q}$ (these indices correspond to the order of the strands near $Q$, not to the labeling of the lines). Turning around the point $Q$ is associated to the braid $\Delta_{Q}^{2}$, which consists on the full-twist of those $m_{Q}$ strands, and straight lines for the other strands. If $\mu_{1}^{Q}, \ldots, \mu_{11}^{Q}$ is a geometric basis of meridians in a vertical line close to $x=Q$, the relations obtained are:

$$
\mu_{j}^{Q}=\left(\mu_{j}^{Q}\right)^{\Delta_{Q}^{2}}, \quad 1 \leq j \leq 11 .
$$

The exponent stands for the geometric action of the braid group on the free group. Any one of above the relations is a consequence of the other ones, but we can be more precise. If 
$\mu(Q)=\prod_{j=i_{Q}+1}^{i_{Q}+m_{Q}} \mu_{j}^{Q}$, then the above relations read as

$$
\mu_{j}^{Q}= \begin{cases}\mu(Q)^{-1} \cdot \mu_{j}^{Q} \cdot \mu(Q) & \text { if } j=i_{Q}+1, \ldots, i_{Q}+m_{Q}, \\ \mu_{j}^{Q} & \text { otherwise. }\end{cases}
$$

Eliminating unnecessary relations, we keep:

$$
\mu_{i_{Q}+j}^{Q}=\mu(Q)^{-1} \cdot \mu_{i_{Q}+j}^{Q} \cdot \mu(Q) \quad \forall j=1, \ldots, m_{Q}-1 .
$$

The wiring diagram provides the global information which allows to deal with all these relations together. Namely there is a braid $\beta_{Q}$ connecting the generic vertical line close to $Q$ and a generic vertical line in left-hand side of the diagram. The global braid is $\alpha_{Q}:=\beta_{Q}^{-1} \cdot \Delta_{Q}^{2} \cdot \beta_{Q}$. This braid produces the relations

$$
\mu_{i}=\mu_{i}^{\alpha_{Q}}, \quad 1 \leq i \leq 11 .
$$

As before, we can reduce these relations as:

$$
\left[\mu_{i_{Q}+j}^{\beta_{Q}}, \mu(Q)^{\beta_{Q}}\right]=1, \quad j=1, \ldots, m_{Q}-1 .
$$

One has the following.

Proposition 4.1. The fundamental group $G^{i}$ admits a presentation

$$
\left.\left\langle\mu_{k}, k \in\{1, \ldots, 11\} \mid 4.4\right\rangle\right\rangle .
$$

Using Proposition 4.1 and the braided wiring diagrams given above the presentations can be obtained. They are coded in Part A.1.

Remark 4.2. The meridian $\mu_{k}$ corresponds to the line $L_{k}$ except for the line $L_{12} \equiv L_{5}^{\prime}$ which corresponds to $\mu_{5}$; note that $L_{5}$ is the line at infinity, and its meridians can be expressed as words in the other ones.

\subsection{Alexander invariant computations and the AI-isomorphism test.}

To avoid the annoying problem that we consider no meridian for the line $L_{5}$, from now on we number the lines with the index of their meridians; the line at infinity (former line $L_{5}$ ) is now the line $L_{0}$.

From the relations induced by (4.4) and the Jacobi relations (3.1) we obtain a presentation for the truncated Alexander invariants $M_{k}^{i}, k=1,2$ (see Definition 3.1 and Example 3.3. Note that $M_{1}^{i}$ depends only on the combinatorics $\mathscr{G}_{91}$. In particular, in our case, $M_{1}^{i}$ is generated by $\left\{x_{j, k}:=x_{L_{j}, L_{k}} \mid 1 \leq j, k \leq 11\right\}$ and relations $\sum_{L_{k}<P} x_{j, k}=0$ for each $L_{j}<P$, and for each $P$ such that $L_{0} \nless P$. We assume the convention that $x_{k, j}=-x_{j, k}$.

This implies that $M_{1}^{i} \cong H_{2}$ via the morphism $x_{j, k} \mapsto x_{L_{j}} \wedge x_{L_{k}}$ as a consequence of 2.1]. We can choose $\mathcal{B} \subset\{(j, k) \mid 1 \leq j<k \leq 11\}$ such that $\left\{x_{j, k} \mid(j, k) \in \mathcal{B}\right\}$ is a basis of $M_{1}^{i}$.

As for $M_{2}^{i}$, the actual presentations of $G^{i}$ and the Jacobi relations 3.1 are in this case required. Note that $\operatorname{rank} M_{2}^{i}=\operatorname{rankgr}{ }^{0} M_{2}^{i}+\operatorname{rankgr}^{1} M_{2}^{i}$. With the relations coming from the 
group we can see that $\left\{\sigma_{h} \cdot x_{j, k} \mid 1 \leq h \leq 11,(j, k) \in \mathcal{B}\right\}$ generate $M_{2}^{i}$, subject to the relations induced by 3.1 .

Let us describe the AI-isomorphism test in a general setting. Assume $\left(G^{1}, h_{1}\right)$ and $\left(G^{2}, h_{2}\right)$ are fundamental groups of two arrangements with the same combinatorics $\mathscr{C}=(\mathcal{L}, \mathcal{P})$ and with their meridian structures. Let $\ell=|\mathcal{L}|-1$ and let $L_{0}, L_{1}, \ldots, L_{\ell}$ be the lines and choose a set $\mathcal{B}$ as above. Assume there is an isomorphism $\varphi$ fitting in the following diagram:

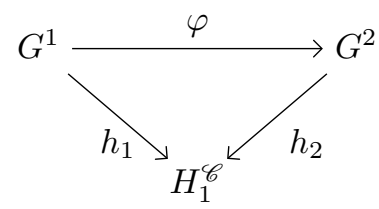

For $1 \leq k \leq \ell$, we write $\mu_{k, i}$ for a generator of $G^{i}$ which is a meridian whose image by $h_{i}$ is $x_{k}:=x_{L_{k}} \in H_{1}^{\mathscr{C}}$. Note that $G^{i}$ is generated by $\mu_{k, i}, 1 \leq k \leq \ell$.

(AI-1) The isomorphism $\varphi$ is determined by $\varphi\left(\mu_{k, 1}\right)=\mu_{k, 2} \cdot g_{k}$, where $g_{k} \in\left(G^{2}\right)^{\prime}$. Note that this does not necessarily mean that $\varphi$ preserves meridians, since $\mu_{k, 1} \cdot g_{k}$ is not necessarily in the same conjugacy class as $\mu_{k, 2}$.

(AI-2) The isomorphism $\varphi$ induces a $\Lambda / \mathfrak{m}_{2}$-isomorphism $\varphi_{*}: M_{2}^{1} \rightarrow M_{2}^{2}$. Let us denote $x_{j, k}^{i}:=$ $\left[\mu_{j, i}, \mu_{k, i}\right] \bmod \Lambda / \mathfrak{m}^{2}$. If no confusion is likely to arise, we will drop the super-index. We have

$$
x_{j, k} \mapsto\left[\mu_{j, 2} \cdot g_{j}, \mu_{k, 2} \cdot g_{k}\right] \bmod \Lambda / \mathfrak{m}^{2} .
$$

(AI-3) Because of Proposition 3.6, only the class of $g_{j}$ in $M_{1}^{2}$ is needed, $\varphi_{*}$ is determined by

$$
g_{j} \stackrel{1}{\equiv} \sum_{(h, k) \in \mathcal{B}} n_{j, h, k} \cdot x_{h, k},
$$

for some $n_{j, h, k} \in \mathbb{Z}$.

(AI-4) Take a relation $R \in G^{1}$. This relation induces a linear equality $R_{1}$ in $M_{2}^{1}$, written in terms of $x_{i, j} \in M_{2}^{1}$ for $1 \leq i<j \leq \ell$. Using (4.6) and 4.7), its image under $\varphi_{*}$ is an equation $R_{2}$ in the module $M_{2}^{2}$, whose unknowns are the variables $n_{j, h, k}$ of (4.7).

(AI-5) One can reduce the equation $R_{2}$ using the relations of $M_{2}^{2}$ to obtain equations in $\operatorname{gr}^{1} M_{2}$.

The existence of solutions for this system is a necessary condition for the existence of such an isomorphism $\varphi$. The process we have outlined above is referred to as the AI-isomorphism test of level 2. If the necessary condition is true, we can use the integer solutions to work in $M_{3}$, and so on. This process at each $M_{k}$ will be referred to as the AI-isomorphism test of level $k$ for the pair $\left[\left(G^{1}, h_{1}\right),\left(G^{2}, h_{2}\right)\right]$.

Proposition 4.3. Under the notation above, the pair $\left[\left(G^{1}, h_{1}\right),\left(G^{2}, h_{2}\right)\right]$ do not pass the AIisomorphism test of level 2 . In other words, there is no isomorphism $\left(G^{1}, h_{1}\right) \rightarrow\left(G^{2}, h_{2}\right)$ inducing the identity $1_{H_{1}^{\mathscr{G}} 91}$.

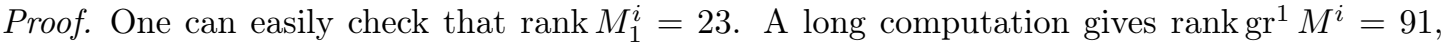
hence each equation $\tilde{R}_{2}$ in $\operatorname{gr}^{1} M^{2}$ induces 91 linear equations; since there are 32 such relations, 
we obtain a linear system of 2912 equations in $23 \times 11=253$ unknowns. After eliminating trivial equations, we have to deal with a linear system of 930 equations with 253 variables. We have attacked this system with Sagemath [24], using pivoting methods where divisions are not allowed.

We obtain that the solutions over $\mathbb{Q}$ is an affine space of dimension 12 , but the smallest ring where solutions exist is $\mathbb{Z}\left[\frac{1}{5}\right]$. In particular, no integer solution exists. These computations, together with the presentation of $M_{1}^{i}$ and $M_{2}^{i}$ took 662.49s of CPU time on an 8 double core Athlon processor computer with 128GB of RAM.

We can apply the same ideas to the groups $G^{4}$ and $G^{1}$. Let us recall that, even though $G^{1}$ and $G^{4}$ are isomorphic, their meridian structures are reversed, i.e. the known isomorphism induces $-1_{H_{1}^{\mathscr{S}}}$.

Proposition 4.4. Under the notation above, the pair $\left[\left(G^{4}, h_{4}\right),\left(G^{2}, h_{2}\right)\right]$ do not pass the AIisomorphism test of level 2 . In other words, there is no isomorphism $\left(G^{4}, h_{4}\right) \rightarrow\left(G^{2}, h_{2}\right)$ inducing the identity $1_{H_{1}^{\varphi_{91}}}$.

Proof. We repeat the above procedure skipping the computations about $M_{2}^{2}$, already done. After 97.69s of CPU time, we obtain similar results as in Proposition 4.4.

With all the above we are ready to prove the main result.

Theorem 4.5. The fundamental groups $G^{1}$ and $G^{2}$ of the complement to the realizations $\mathcal{A}^{\xi}$ and $\mathcal{A}^{\xi^{2}}$ of the combinatorics $\mathscr{G}_{91}$ are not isomorphic.

Proof. Consider the presentations of $G^{i}$ given in $\$ 1$, which provide meridian structures in a natural way. Assume $\varphi: G^{1} \rightarrow G^{2}$ is an arbitrary isomorphism. By Proposition 2.8, $\mathscr{G}_{91}$ is homologically rigid and Aut $\mathscr{G}_{91}=1 \mathscr{\mathscr { G }}_{91}$. As a consequence, $\varphi$ induces $\varphi_{*}= \pm 1_{H_{1}^{\mathscr{G}_{11}}}$, see Remark 2.2. If $\varphi_{*}=1_{H_{1}^{\mathscr{G}_{91}}}$, then Proposition 4.3 results in a contradiction. Assume $\varphi_{*}=-1_{H_{1}^{\mathscr{S}_{91}}}$. Note that, as mentioned at the beginning of 4.1, the conjugation morphism $\psi$ induces an automorphism of groups whose associated morphism $\psi_{*}$ equals $-1_{H_{\mathscr{G}_{91}}^{1}}$. Composing $\psi \circ \varphi$ one obtains an isomorphism $\varphi \circ \psi: G^{4} \rightarrow G^{2}$ satisfying $(\varphi \circ \psi)_{*}=1_{H_{\mathscr{G}_{91}}^{1}}$, which can be disregarded using Proposition 4.4. This ends the proof.

\section{REFERENCES}

[1] H. Abelson, Topologically distinct conjugate varieties with finite fundamental group, Topology 13 (1974), $161-176$.

[2] E. Artal, Sur les couples de Zariski, J. Algebraic Geom. 3 (1994), no. 2, 223-247.

[3] _ Topology of arrangements and position of singularities, Ann. Fac. Sci. Toulouse Math. (6) 23 (2014), no. 2, 223-265.

[4] E. Artal, J. Carmona, and J.I. Cogolludo-Agustín, Braid monodromy and topology of plane curves, Duke Math. J. 118 (2003), no. 2, 261-278.

[5] __ Effective invariants of braid monodromy, Trans. Amer. Math. Soc. 359 (2007), no. 1, 165-183.

[6] E. Artal, J. Carmona, J.I. Cogolludo-Agustín, and M.Á. Marco, Topology and combinatorics of real line arrangements, Compos. Math. 141 (2005), no. 6, 1578-1588. 
[7] _ Invariants of combinatorial line arrangements and Rybnikov's example, Singularity theory and its applications (S. Izumiya, G. Ishikawa, H. Tokunaga, I. Shimada, and T. Sano, eds.), Advanced Studies in Pure Mathematics, vol. 43, Mathematical Society of Japan, Tokyo, 2007.

[8] E. Artal, J.I. Cogolludo-Agustín, and H. Tokunaga, A survey on Zariski pairs, Algebraic geometry in East Asia-Hanoi 2005, Adv. Stud. Pure Math., vol. 50, Math. Soc. Japan, Tokyo, 2008, pp. 1-100.

[9] E. Artal, V. Florens, and B. Guerville-Ballé, A topological invariant of line arrangements, Preprint available at arXiv:1407.3387 [math.GT], 2014.

[10] F. Charles, Conjugate varieties with distinct real cohomology algebras, J. Reine Angew. Math. 630 (2009), $125-139$.

[11] D. C. Cohen and A. I. Suciu, The braid monodromy of plane algebraic curves and hyperplane arrangements, Comment. Math. Helv. 72 (1997), no. 2, 285-315.

[12] A.I. Degtyarëv, On deformations of singular plane sextics, J. Algebraic Geom. 17 (2008), no. 1, 101-135.

[13] M. Falk and S. Yuzvinsky, Multinets, resonance varieties, and pencils of plane curves, Compos. Math. 143 (2007), no. 4, 1069-1088.

[14] V. Florens, B. Guerville-Ballé, and M.Á. Marco, On complex line arrangements and their boundary manifolds, Math. Proc. Cambridge Philos. Soc. 159 (2015), no. 2, 189-205.

[15] G. González-Diez and D. Torres-Teigell, Non-homeomorphic Galois conjugate Beauville structures on $\operatorname{PSL}(2, p)$, Adv. Math. 229 (2012), no. 6, 3096-3122.

[16] B. Guerville-Ballé, Zariski pairs of line arrangements with twelve lines, Geometry \& Topology 20 (2016), $537-553$.

[17] M.Á. Marco, A description of the resonance variety of a line combinatorics via combinatorial pencils, Graphs Combin. 25 (2009), no. 4, 469-488.

[18] P. Orlik and L. Solomon, Combinatorics and topology of complements of hyperplanes, Invent. Math. 56 (1980), no. 2, 167-189.

[19] C. S. Rajan, An example of non-homeomorphic conjugate varieties, Math. Res. Lett. 18 (2011), no. 5, 937-942.

[20] G. Rybnikov, On the fundamental group of the complement of a complex hyperplane arrangement, Funct. Anal. Appl. 45 (2011), 137-148, Preprint available at arXiv:math.AG/9805056.

[21] J.-P. Serre, Exemples de variétés projectives conjuguées non homéomorphes, C. R. Acad. Sci. Paris Sér. I Math. 258 (1964), 4194-4196.

[22] I. Shimada, On arithmetic Zariski pairs in degree 6, Adv. Geom. 8 (2008), no. 2, 205-225.

[23] _ Non-homeomorphic conjugate complex varieties, Singularities-Niigata-Toyama 2007, Adv. Stud. Pure Math., vol. 56, Math. Soc. Japan, Tokyo, 2009, pp. 285-301.

[24] W.A. Stein et al., Sage Mathematics Software (Version 6.7), The Sage Development Team, 2015, http://www. sagemath.org.

[25] S. Yuzvinsky, A new bound on the number of special fibers in a pencil of curves, Proc. Amer. Math. Soc. 137 (2009), no. 5, 1641-1648.

\section{Appendix A. Sagemath code}

Part A.1. Coding the wiring diagrams

The following code presents the two wiring diagrams, and the groups $\mathbb{B}_{n}, \mathbb{F}_{n}$, for $n=11$. The lines and their meridians are labelled as $k$ for $\mu_{k}$ and $L_{k}\left(k \neq 5\right.$ since no meridian of $L_{5}$ is needed) and $k=5$ refers to $\mu_{5}$, the meridian of $L_{5}^{\prime} \equiv L_{12}$. For further use, we introduce the permutations orden \pm relating the order at the base vertical line of the wiring diagram, e.g. the sequence $[1,4,9,5,10,11,2,7,8,6,3]$ reflects the order of the lines in the wiring diagram shown 
in Figure 2 from top to bottom. Since we are interested in a generic wiring diagram, this will be obtained by slightly rotating the vertical lines in the counterclockwise direction.

The lists wiring \pm have as many entries as singular crossings $c_{n}$ of the wiring diagram. Each entry contains two lists: the first one encodes the braid -if any- described by the wiring diagram from the previous singular crossing $c_{n-1}$ to $c_{n}$ and the second list enumerates the lines involved in the crossing. For the lines involved in each crossing, the labels $k$ refer to the line $L_{k}$ (for $k \neq 5$ and $L_{5}^{\prime}=L_{12}$ for $k=5$ ). As for the braid, the numbers refer to the generators of the braid group counting the strings from top to bottom, and the sign refers to the sign of the crossing.

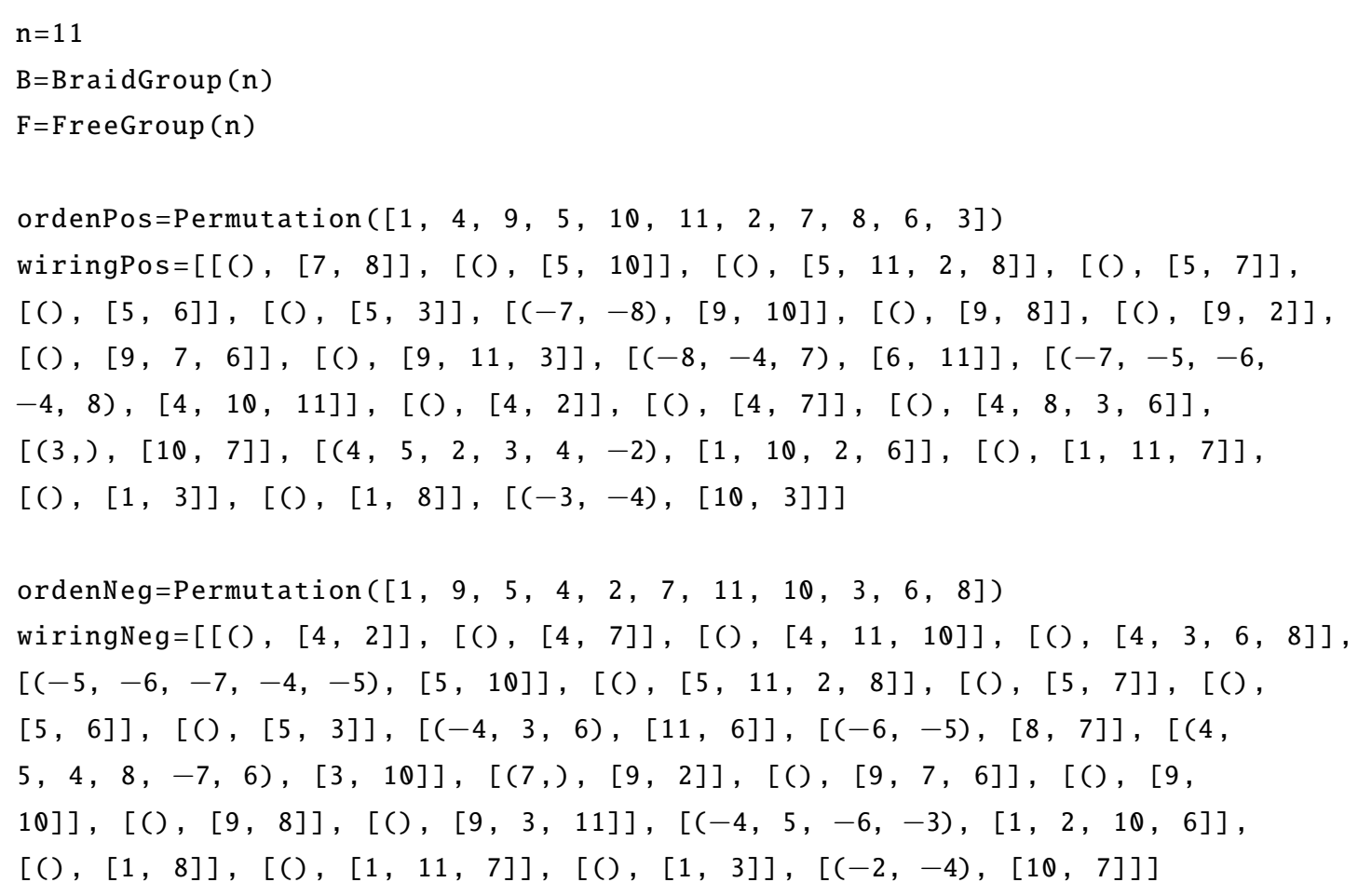

Part A.2. Core of the computation

Step 1. We start constructing the presentations of the groups using the function in (S3). We construct the list inc representing a family $\mathcal{B} \subset\{(i, j) \mid 1 \leq i<n, i<j \leq n\}$ such that $\left\{x_{i, j} \mid(i, j) \in \mathcal{B}\right\}$ is a basis of the truncated Alexander invariant $M_{1}$.

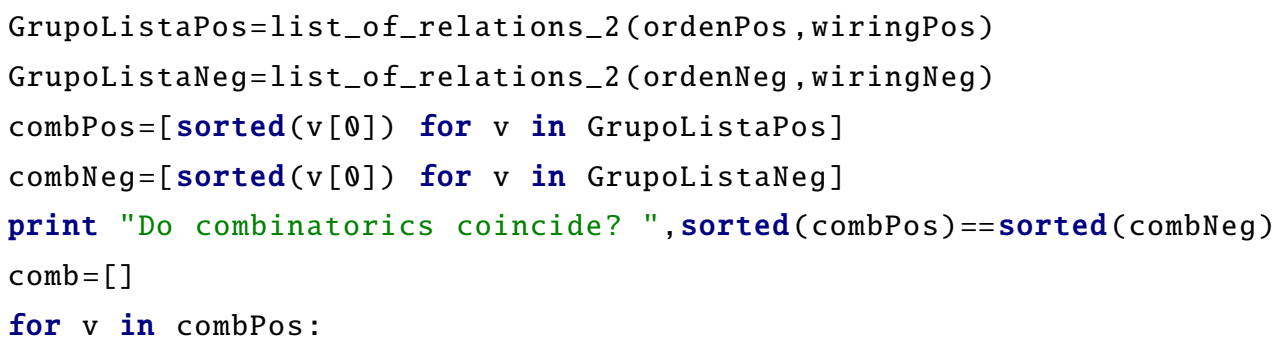




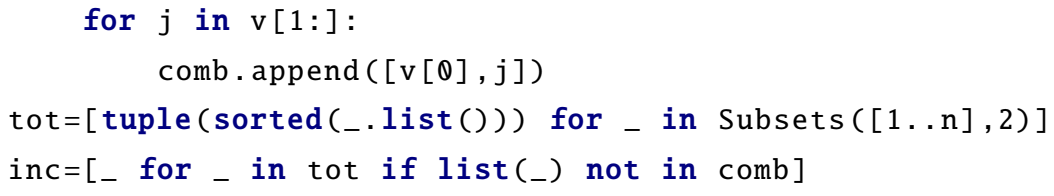

Step 2. We construct several rings. We start with $S:=\mathbb{Z}\left[x_{k, i, j} \mid 1 \leq k \leq n,(i, j) \in \mathcal{B}\right]$. The variables of this ring are the unknowns of the final linear system. We define a dictionnary YY to track the variables of $S$ using the the triples $(k, i, j)$. The ring LR is the Laurent polynomial ring $\mathbb{Z}\left[t_{1}^{ \pm 1}, \ldots, t_{n}^{ \pm 1}\right]=\mathbb{Z}[H]$ and LRv is $S\left[t_{1}^{ \pm 1} \ldots, t_{n}^{ \pm 1}\right]=S[H]$ while $R:=\mathbb{Z}\left[t_{1}, \ldots, t_{n}\right], T:=$ $S\left[\left[s_{1}, \ldots, s_{n}\right]\right]$ and $T_{0}:=\mathbb{Z}\left[\left[s_{1}, \ldots, s_{n}\right]\right]$. The dictionnaries dic and dicv realize the substitution $t_{i} \mapsto 1+s_{i}$ in each ring.

$\mathrm{xx}=\operatorname{var}\left(\left[{ }^{\prime} \mathrm{v} \% \mathrm{da} \% \mathrm{db} \% \mathrm{~d} \mathrm{~d}^{\prime}(\mathrm{k}, \mathrm{i}, j)\right.\right.$ for $\mathrm{k}$ in $[1 \ldots \mathrm{n}]$ for $(i, j)$ in inc $\left.]\right)$

$\mathrm{S}=$ PolynomialRing $(Z Z, x x)$

$\mathrm{YY}=\{\}$

for $k$ in $[1 \ldots n]$ :

for 1 in range(len(inc)):

$\mathrm{YY}[\mathrm{k}$, inc $[1][0]$, inc $[1][1]]=\mathrm{S} \cdot \operatorname{gen}((\mathrm{k}-1) * \operatorname{len}(\mathrm{inc})+1)$

LR=LaurentPolynomialRing (ZZ, 't', n)

LRV $=$ LR . change_ring (S)

$\mathrm{R}=\mathrm{LR}$. polynomial_ring ()

$\mathrm{T}=$ PowerSeriesRing ( $\mathrm{S}$, ' $\mathrm{S}$ ' , num_gens=n, default_prec=2)

$\mathrm{T} Q=\mathrm{T}$. change_ring (ZZ)

HomLRT $\theta=R \cdot h o m([1+v$ for $v$ in $T \theta \cdot \operatorname{gens}()]$, codomain $=T \theta$, check=True $)$

dic $=\{v: \operatorname{HomLRTQ}(\mathrm{v})$ for $\mathrm{v}$ in LR.gens ()$\}$

$\operatorname{dicv}=\{v: \operatorname{HomLRT} Q(v)$. change_ring $(S)$ for $v$ in LRv.gens ()$\}$

Step 3. We create the free module $M$ with basis $x_{i, j}$ and base ring LR. The dictionnary XX expresses any $\left[x_{i}^{ \pm 1}, x_{j}^{ \pm 1}\right]$ in the module.

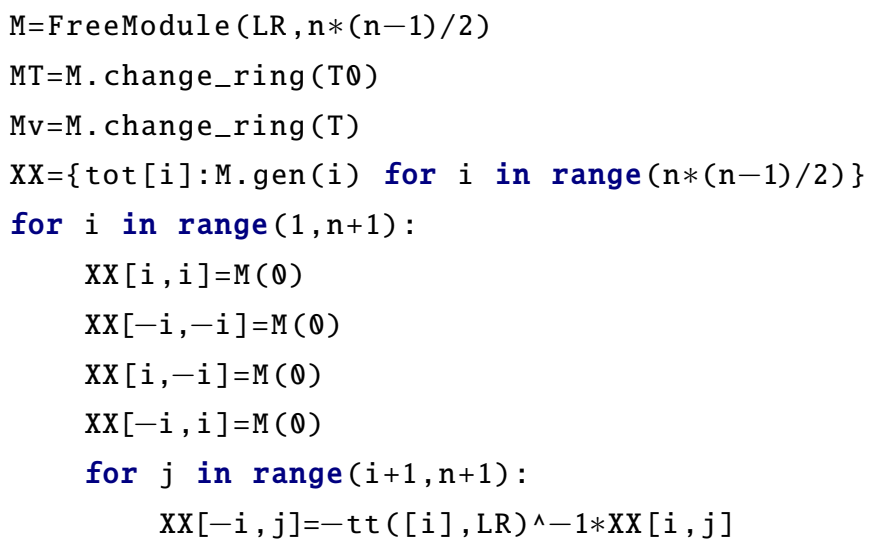




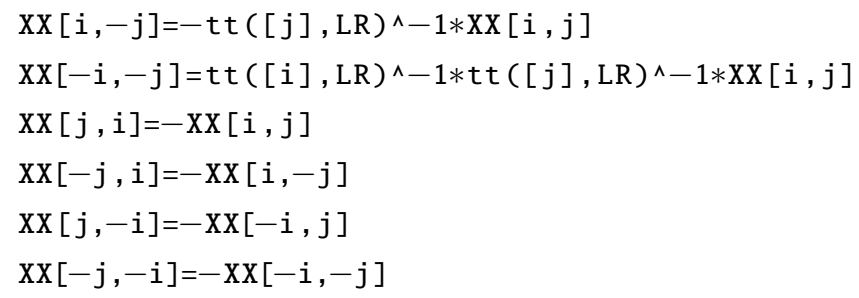

Step 4. We translate the relations of both groups as elements of the module $M$. We write down both sets of relations as matrices (the number of rows is the number of relations and columns related to all the $x_{i, j}$ and we translate them into the series ring. The matrix SustNeg expresses all the $x_{i, j}$ in terms of those such that $(i, j) \in \mathcal{B}$.

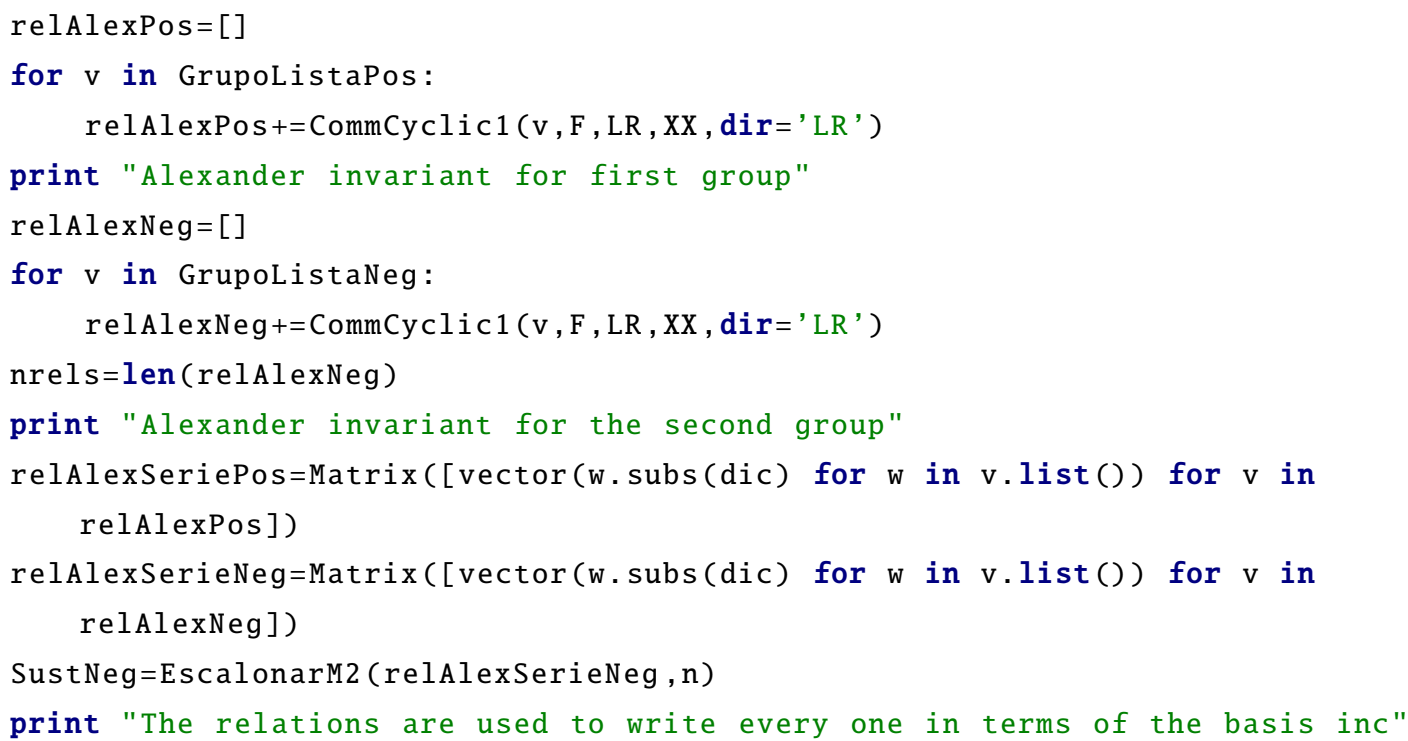

Step 5. The main point is to test if there is a homomorphism $\varphi: G_{1} \rightarrow G_{2}$ such that $x_{k} \in G_{1}$ is sent to $x_{k} \prod_{(i, j) \in \mathcal{B}}\left[x_{i}, x_{j}\right]^{x_{k, i, j}} \bmod \tilde{\gamma}_{4}(G)$. We express the image of $\left[x_{i}, x_{j}\right]$ as an element in $M \otimes T$ (this is by far the most long computation!). With this data, we compute the image of the relations of $G_{1}$, which will be now words in the truncated Alexander invariant $M_{2} \otimes T$.

We write these elements only in terms of $x_{i, j},(i, j) \in \mathcal{B}$. The next step is express the relations as linear combination of $s_{k} x_{i, j}, 1 \leq k \leq n$ and $(i, j) \in \mathcal{B}$, with coefficients in $S$.

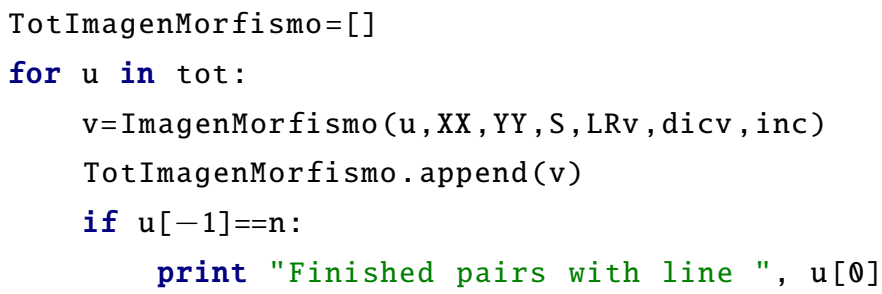




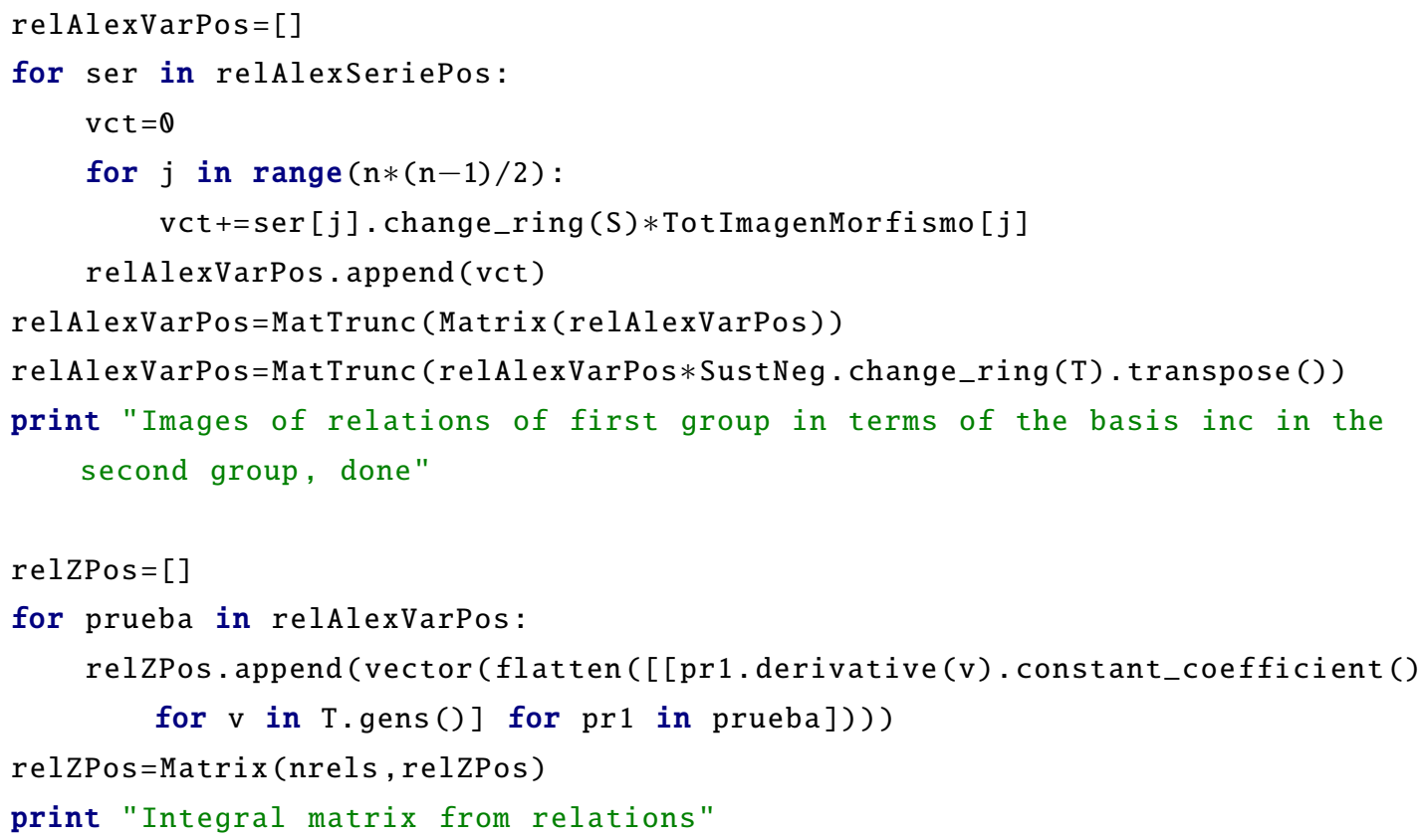

Step 6. In order to compute $M_{2}$ we need to add the relations given by Jacobi identities as $\mathbb{Z}$-linear combinations in $\left\{s_{k} x_{i, j} \mid 1 \leq k \leq n,(i, j) \in \mathcal{B}\right\}$. We quotient the previous free module by this relation. In this case we obtain a free abelian module; we pass from $23 \times 11=253$ relations to 91 .

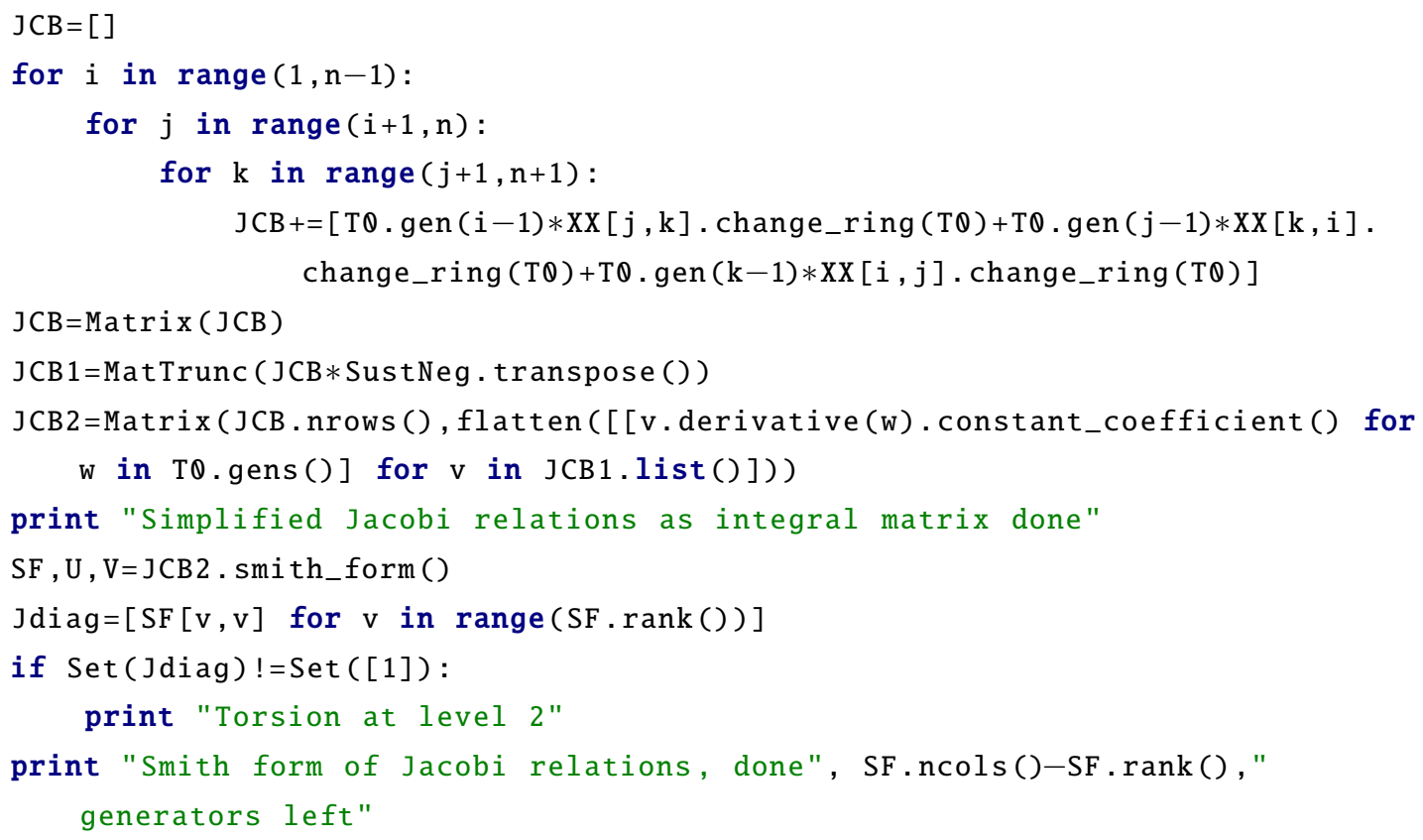


Step 7. We write down the relations taking into account Jacobi relations. The equations are all the coefficients in the basis. We solve the linear system of 930 equations and 253 unknowns. The system has relations over $\mathbb{Q}$ but not over $\mathbb{Z}$ (the smallest ring is $\mathbb{Z}\left[\frac{1}{5}\right]$ ).

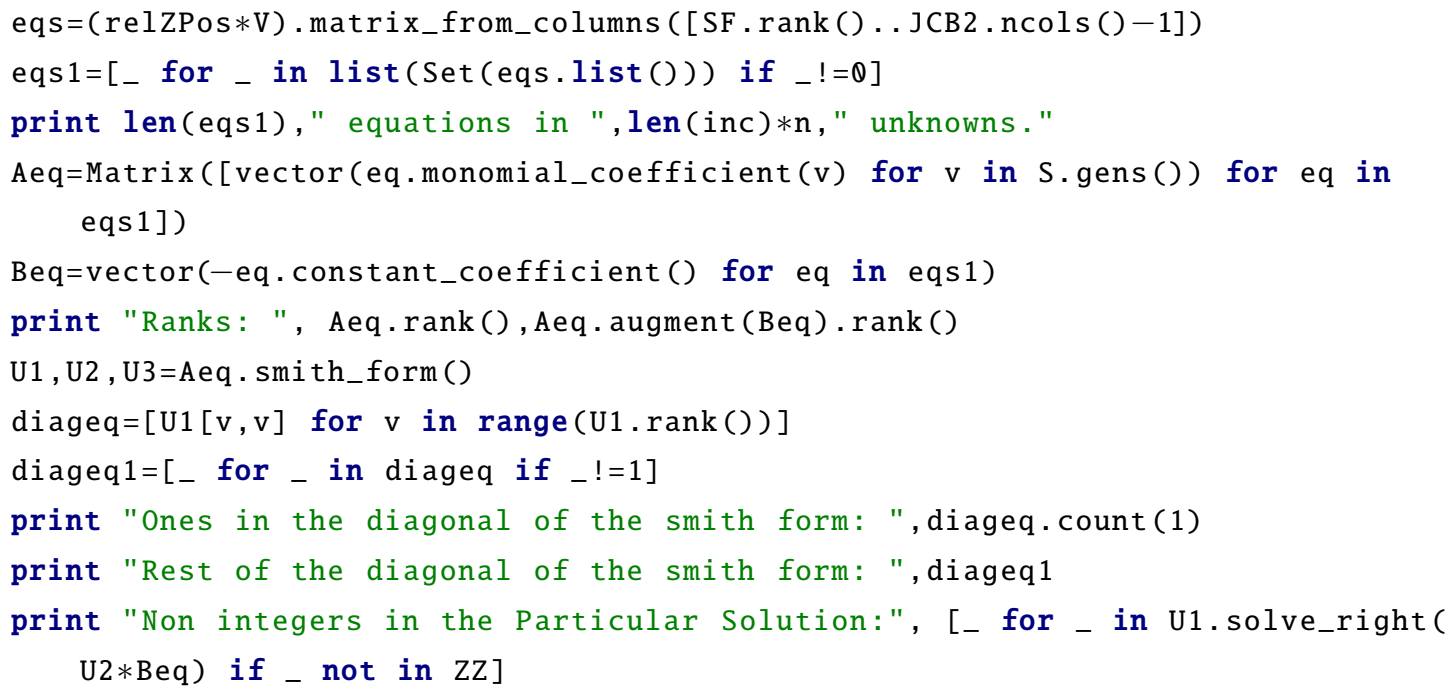

Part A.3. Some auxiliar functions.

(S1) Delta

Input: A list of consecutive numbers $[i, \ldots, j]$ and a braid group.

Output: The half-twist braid involving the strands $i, \ldots, j$.

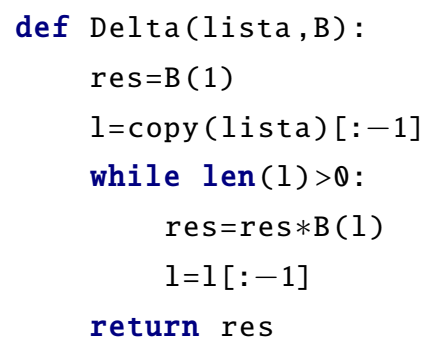

(S2) clean_conj

Input: A list of numbers $[i, \ldots, j]$ and a free group. The list of numbers represents the Tietze representation of an element $x$ in the free group (with generators $g_{j}$ ).

Output: It returns None if $x$ is not conjugate to a generator. If it is, it returns a list $\left[[i],\left[j_{1}, \ldots, j_{r}\right]\right]$ such that $x=x_{i}^{x_{j_{1}} \cdot \ldots \cdot x_{j_{r}}}$.

def clean_conj(lista,F):

$\mathrm{n}=\operatorname{len}(\mathrm{lista})$ 


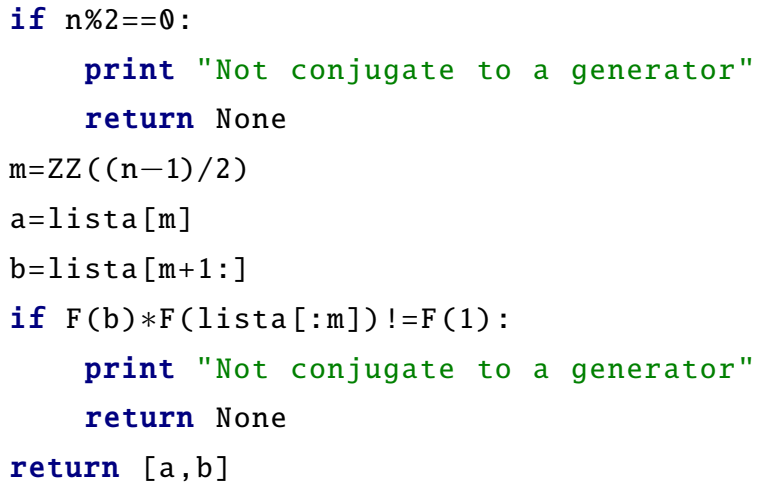

(S3) list_of_relations_2

Input: A permutation to indicate the order of the lines in the beginning and a wiring diagram, see Part A.1. For each crossing we give a list with two members: the braid from the previous crossing, and the lines in the crossing. We assume a generic wiring diagram.

Output: It returns the presentation of the group, as a list of elements with two entries; the first one is a list $\left[i_{1}, \ldots, i_{r}\right], r \geq 2$; the second one is a list of $r$ lists, which represent the Tietze representation of words $w_{1}, \ldots, w_{r}$. This element means that $x_{i_{1}}^{w_{1}} \cdot \ldots \cdot x_{i_{r}}^{w_{r}}$ commutes with $x_{i_{1}}^{w_{1}}, \ldots, x_{i_{r}}^{w_{r}}$ (note that in other papers the reversed product is used).

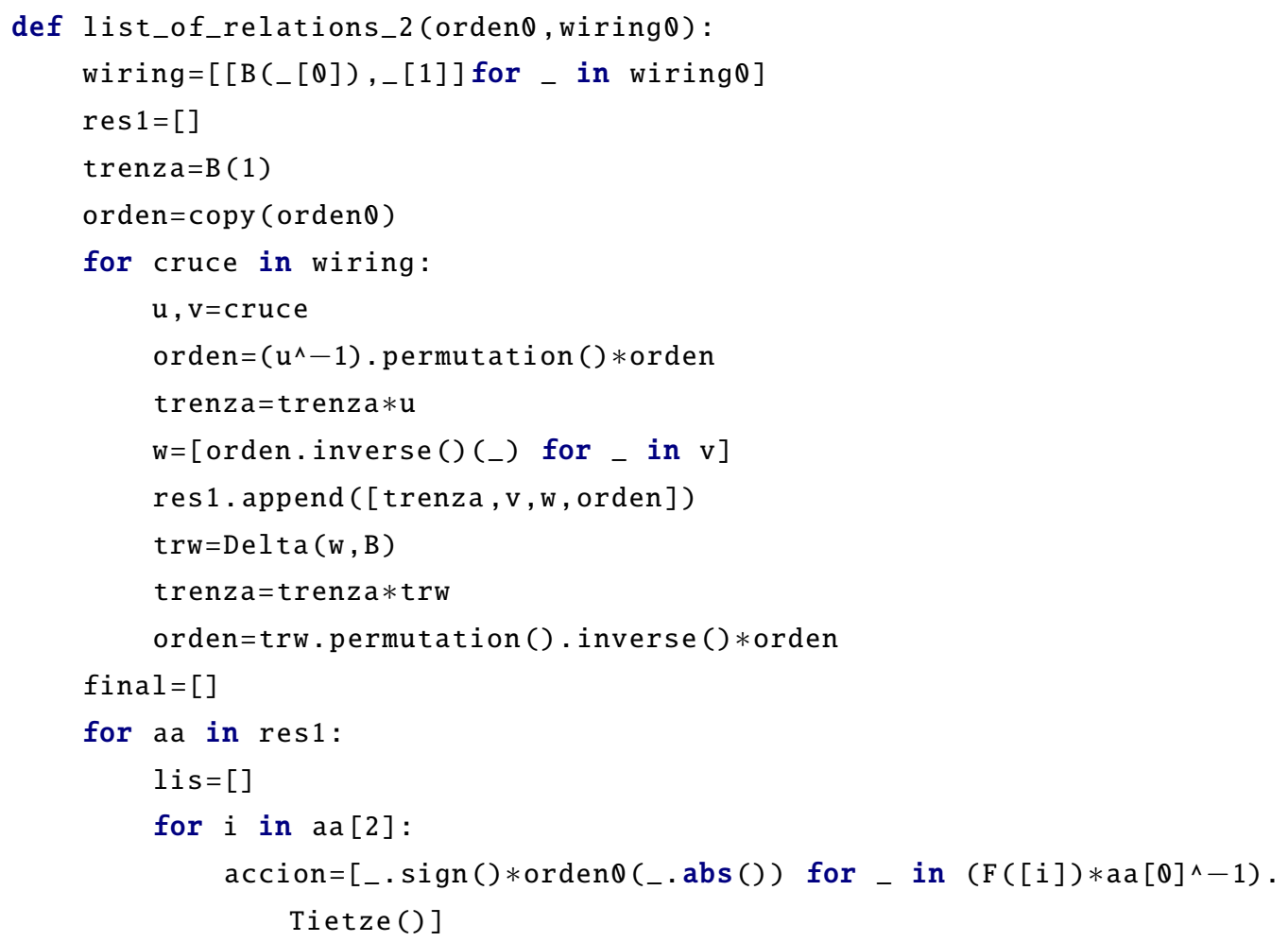




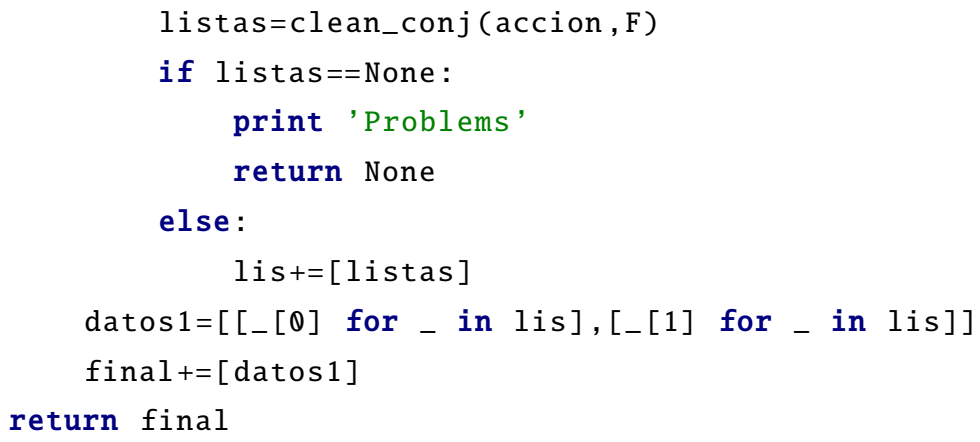

(S4) CommProd1

Input: A number $a \in\{1, \ldots, n\}$, a list representing a element $x$ in the free group, the free group $\mathbb{F}_{n}$ and the ring of Laurent polynomials in $n$ variables and a dictionnary $\mathrm{X}$ associating to each $[i, j]$ an element in the free module over the Laurent ring polynomial.

Output: The element $\left[g_{a}, x\right]$ written as an element of the Alexander invariant $M$.

$\operatorname{def} \operatorname{CommProd} 1(\mathrm{a}, 11, \mathrm{~F}, \mathrm{LR}, \mathrm{XX})$ :

$111=$ list (F(11). Tietze())

if $\operatorname{len}(111)==0$ :

return $M(\theta)$

return $\mathrm{XX}[\mathrm{a}, 111[0]]+\mathrm{tt}([111[0]], \mathrm{LR}) * \operatorname{CommProd} 1(\mathrm{a}, 111[1:], \mathrm{F}, \mathrm{LR}, \mathrm{XX})$

(S5) CommCyclic1

Input: A list $L$, like the output of list_of_relations_2, the free group $\mathbb{F}_{n}$ and the ring of Laurent polynomials in $n$ variables, a dictionnary $\mathrm{X}$ associating to each $[i, j]$ an element in the free module over the Laurent ring polynomial and a variable indicating the direction of the product yielding the local central element (set by default to RightLeft).

Output: The list of elements in the Alexander invariant induced by the relations given by $L$.

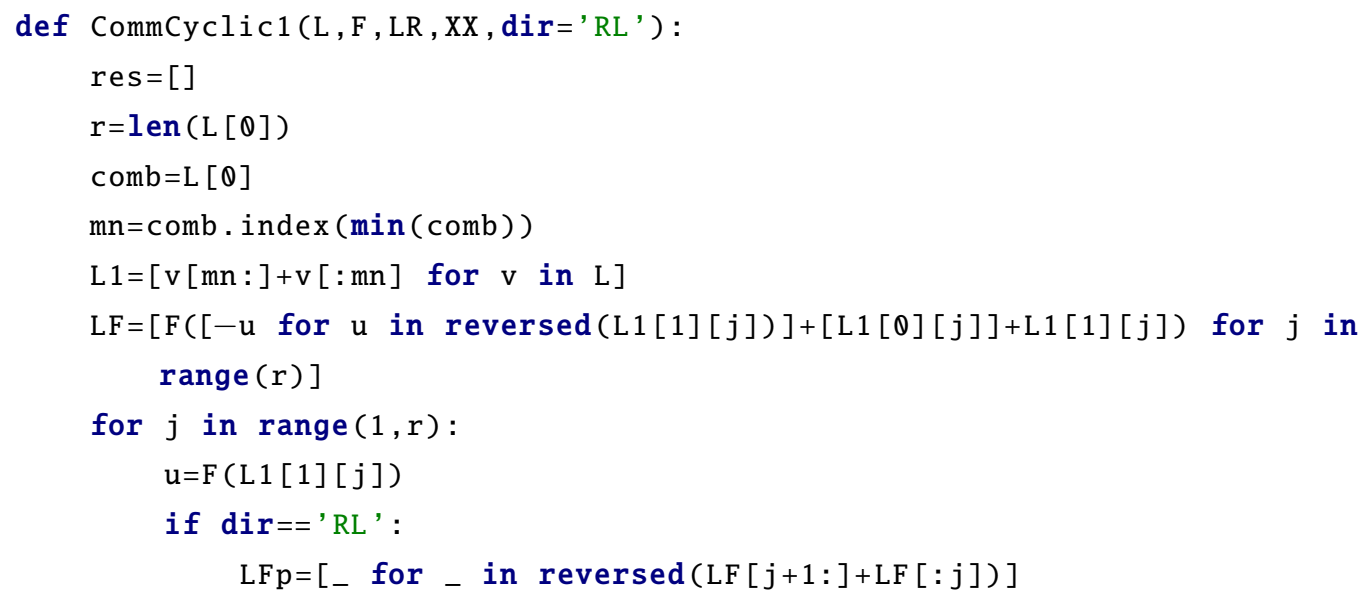




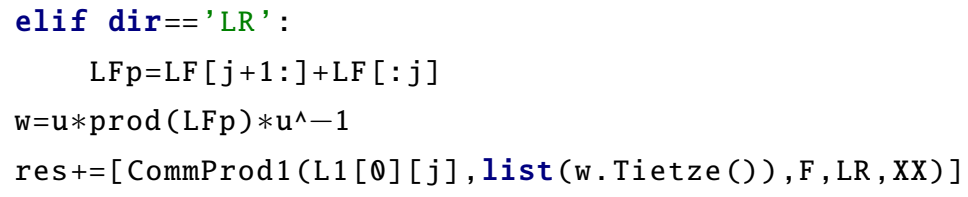

(S6) tt

Input: A list of non-zero integers and a ring of Laurent polynomials in $n$ variables.

Output: The monomial defined by the list.

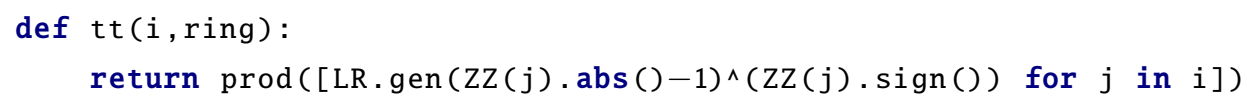

(S7) MatTrunc

Input: A matrix with coefficient in a power series ring.

Output: The matrix where each entry has been truncated to level 2 .

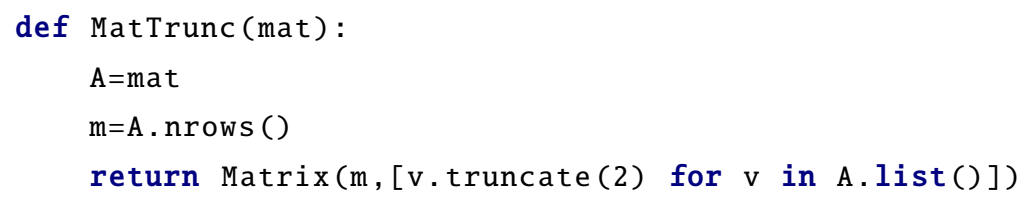

(S8) EscalonarM2

Input: A matrix over the power series ring representing a list of homogeneous linear equations with unknowns $x_{i, j}$ and the number of lines $n$.

Output: Let $\mathcal{B}$ as in Step 1, each column of this matrix is the expression of the corresponding $x_{i, j}$ in terms of the elements $x_{k, l},(k, l) \in \mathcal{B}$ in the Alexander invariant over the power series ring.

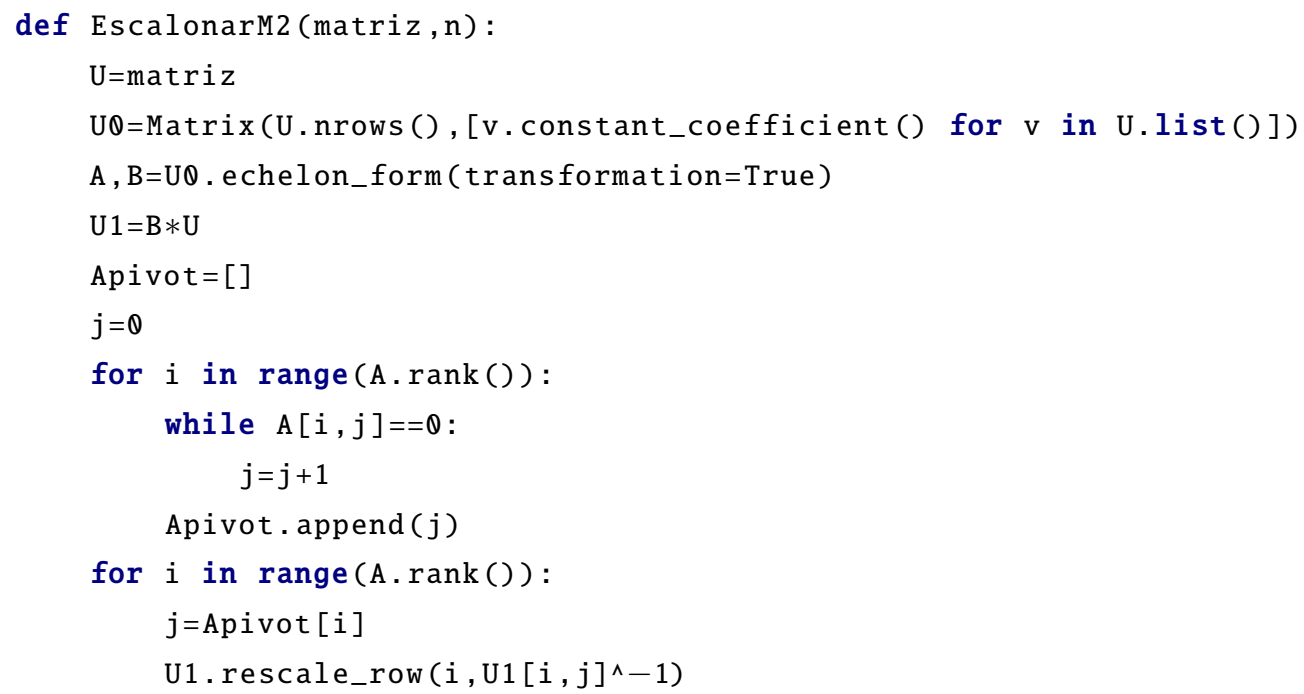




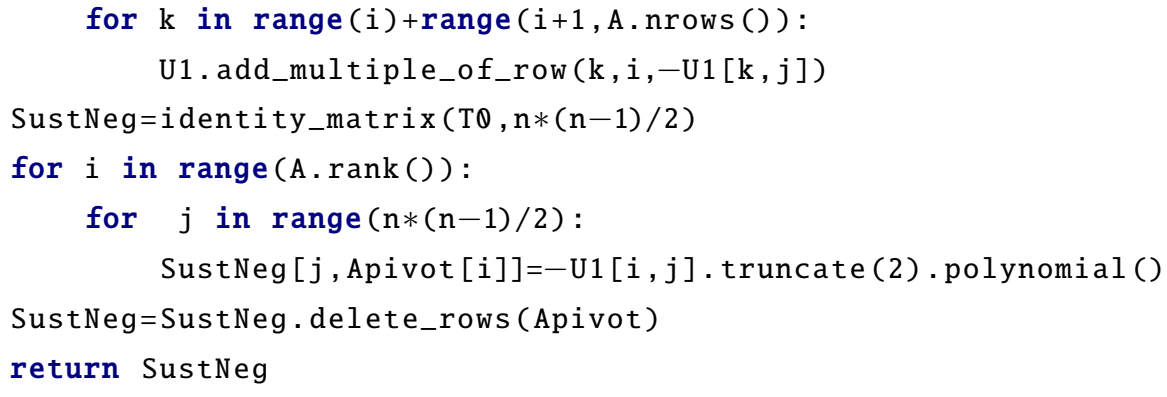

(S9) ImagenMorfismo

Input: $\mathrm{A}$ list $[i, j]$ representing the commutator $x_{i, j}$, a dictionnary $\mathrm{XX}$ associating to each $[i, j]$ an element in the free module over the Laurent ring polynomial, a dictionnary YY associating to each $[k, i, j]$ the unknown $x_{k, i, j}$, representing $\left[x_{k},\left[x_{i}, x_{j}\right]\right] \equiv\left(t_{k}-1\right) x_{i, j} \equiv$ $s_{k} x_{i, j}, S$ is a polynomial ring, LRv is the Laurent Ring with coefficients in the unknowns, dicv is the evaluation $t_{i} \mapsto 1+s_{i}$ and inc is as in Step 1

Output: We consider the group of Step 5. This function uses it to express the image of

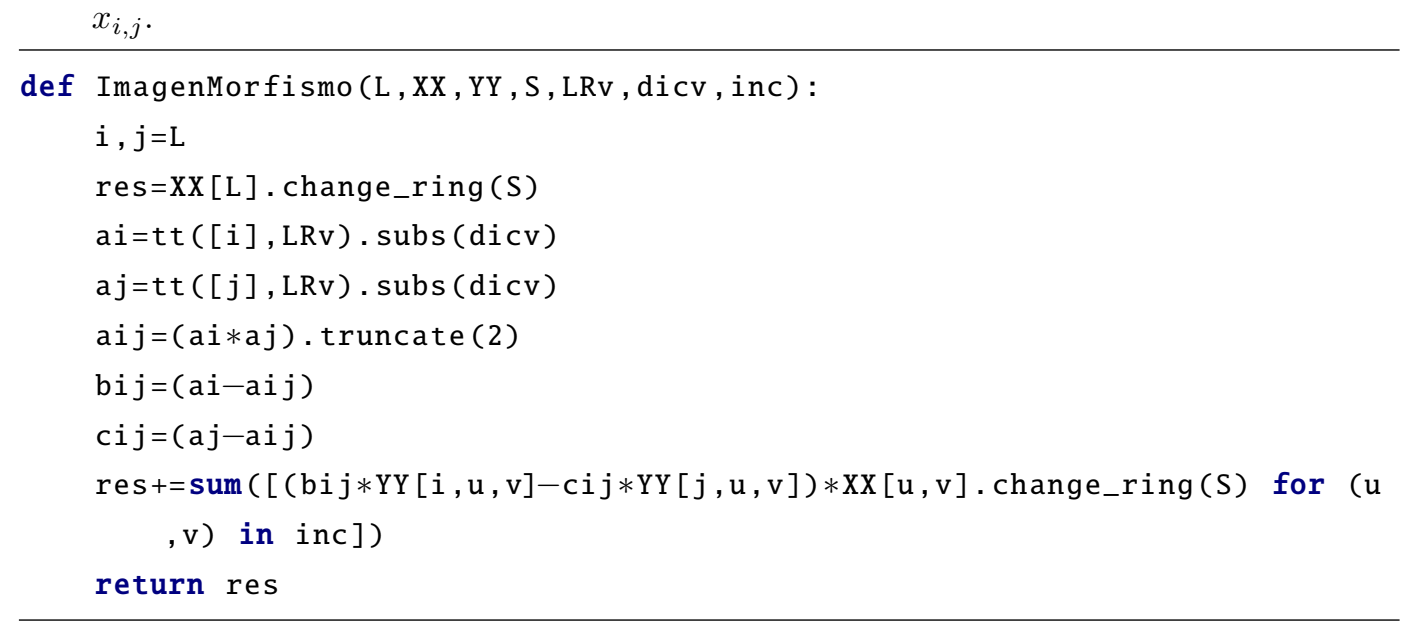

Departamento de Matemáticas, iUma, Universidad de Zaragoza, C. Pedro Cerbuna 12, 50009 Zaragoza, Spain

E-mail address: artal@unizar.es

Departamento de Matemáticas, iUma, Universidad de Zaragoza, C. Pedro Cerbuna 12, 50009 ZARAGOZA, Spain

E-mail address: jicogo@unizar.es

Department of mathematics, Tokyo Gakugei University, Koganei-shi, 184-8501 Tokyo, Japan

E-mail address: benoit.guerville-balle@math.cnrs.fr

Departamento de Matemáticas, iUma, Universidad de Zaragoza, C. Pedro Cerbuna 12, 50009 Zaragoza, Spain

E-mail address: mmarco@unizar.es 